\title{
A LINEAR QUADRATIC PROBLEM WITH UNBOUNDED CONTROL IN HILBERT SPACES*
}

\author{
HANZHONG Wu AND XUNJing Li \\ Institute of Mathematics, Fudan University, Shanghai 200433, China
}

(Submitted by: Viorel Barbu)

\begin{abstract}
This paper considers optimal control problems for an abstract linear system with a quadratic cost functional in a Hilbert space on a fixed time interval $[0, T], 0<T<+\infty$. The controls are unbounded and the state weight operators are indefinite and not necessarily smoothing. Some new inequalities are established and applied to prove that the optimal control and the optimal trajectory are continuous. The equivalences among the solvability of the LQ problems, the two-point boundary value problem and the Fredholm integral equation are proved and the state feedback representation for the optimal control is also given in terms of the solution to the Fredholm integral equation. Finally, we derive the closed-loop synthesis of the optimal control via the solution to the Riccati integral equation which exists under some mild conditions. An explanatory example is given at the end. The results are complementary to those of $[15,16]$ and [17].
\end{abstract}

1. Introduction. Linear quadratic, (LQ) for short, optimal control problem with bounded control in Hilbert spaces was studied by Lions [18], Curtain-Pritchard [5], Balakrishnan [1] and other authors in 1970s. Since 1980s, Lasiecka and Triggiani systematically studied the LQ problems for parabolic, hyperbolic, and other types of partial differential equations with boundary controls. Riccati equation is an important tool in studying the LQ problems, [15] gives an excellent survey in this direction. See Flandoli $[8,9,10]$, Da Prato and Ichikawa [7] and Da Prato [6] as well as Bensoussan, Da Prato, Delfour and Mitter [3] also, for a direct study of the Riccati equation.

Received for publication September 1998.

*This work is partially supported by the National Key Project of China, the National Nature Science Foundation of China, NSF of the Chinese Ministry of Educations and Lab. of Math. for Nonlinear Sciences at Fudan University.

AMS Subject Classifications: 49N10, 93C25. 
Let both $X$ and $U$ be real Hilbert spaces, $-A$ generate an analytic semigroup $e^{-A t}$ on $X$ with $0 \in \rho(A)$, and the fractional power $A^{\gamma}$ be defined for all $\gamma \in \mathbb{R}$. Then, for any $\gamma>0$, there exists a $c_{\gamma}>0$ such that ( see [21] )

$$
\left\{\begin{array}{l}
\left\|A^{\gamma} e^{-A t}\right\| \leq c_{\gamma} t^{-\gamma}, \quad t>0, \quad \text { for any } \gamma>0, \\
\left\|\left(I-e^{-A t}\right) x\right\| \leq c_{\gamma} t^{\gamma}\left\|A^{\gamma} x\right\|, \quad \forall x \in D\left(A^{\gamma}\right), \quad t>0 .
\end{array}\right.
$$

Denote the adjoint of $A$ by $A^{*}$. Then all the above properties of $A$ also hold for $A^{*}$. We assume (1.1) holds for $A^{*}$ and $\left(A^{*}\right)^{\gamma}$ with the same constant $c_{\gamma}$.

In this paper, we consider the following abstract control system

$$
y(t)=e^{-A t} x+\int_{0}^{t} A^{\alpha} e^{-A(t-s)} B u(s) d s \quad(0 \leq \alpha<1),
$$

where $B \in \mathcal{L}(U, X), x \in X$ and $u(\cdot) \in L^{2}(0, T ; U)$. This is the class with hypothesis (H1) in [15] and the one discussed in [16, 17]. For any $x \in X$ and $u(\cdot) \in L^{2}(0, T ; U)$, the solution $y$ to $(1.2)$ is in $L^{2}(0, T ; X)$ by Lemma 1.1 in $\left[17\right.$, p.363]. For any $0 \leq a<b, 0 \leq \gamma<1$ and $z(\cdot) \in L^{2}(a, b ; X)$, it holds with the same constant $c_{\gamma}$ in (1.1) that

$$
\left\|\int_{a} A^{\gamma} e^{-A(\cdot-s)} z(s) d s\right\|_{L^{2}(a, b ; X)} \leq \frac{c_{\gamma}(b-a)^{1-\gamma}}{1-\gamma}\|z(\cdot)\|_{L^{2}(a, b ; X)} .
$$

The abstract setting (1.2) is the mild form of the abstract differential equation

$$
y^{\prime}=-A y+A^{\alpha} B u \quad \text { on }\left[D\left(A^{*}\right)\right]^{\prime}, \quad y(0)=x \in X,
$$

where $D\left(A^{*}\right)$ is the Banach space under the graph norm associated with $A^{*}$ whose dual is denoted by $\left[D\left(A^{*}\right)\right]^{\prime}$. By Corollary 2.1 in $[2$, p.165], for $u(\cdot) \in$ $L^{2}(0, T ; U),(1.4)$ admits a unique weak solution $y$ in $L^{2}(0, T ; X)$, in the sense that (see Definition 3.1 and Remark 3.2 in [2, p.64]), $y \in L^{2}(0, T ; X)$, for all $z \in D\left(A^{*}\right),\langle z, y(\cdot)\rangle_{X} \in W^{1,2}(0, T)$, and for almost all $t \in[0, T]$ and $z \in D\left(A^{*}\right)$

$$
\frac{d}{d t}\langle z, y(t)\rangle=-\left\langle A^{*} z, y(t)\right\rangle+\left\langle\left(A^{*}\right)^{\alpha} z, B u(t)\right\rangle, \quad y(0)=x .
$$

Therefore, (1.2) is well-posed on $X$ as the mild version of (1.4). Meanwhile, it is well-known (see [15-17], etc.) that in the case of second-order parabolic 
equation defined on a bounded domain $\Omega \subset R^{n}$, the relevant values of the constant $\alpha$ are as follows: $\alpha=\frac{3}{4}+\varepsilon, \forall \varepsilon>0$, for Dirichlet boundary control with $U=L^{2}(\partial \Omega), X=L^{2}(\Omega) ; \alpha=\frac{1}{4}+\varepsilon$, for Neumann boundary control with $U=L^{2}(\partial \Omega), X=L^{2}(\Omega)$; and $\frac{n}{4}<\alpha<1$, for pointwise control with $U=H^{-2 \alpha}(\Omega), X=L^{2}(\Omega)$.

With the dynamics (1.2), we define the following quadratic cost functional over a fixed time interval $[0, T], 0<T<+\infty$ :

$$
J(x ; u(\cdot))=\left\langle Q_{1} y(T), y(T)\right\rangle+\int_{0}^{T}\{\langle Q y(t), y(t)\rangle+\langle R u(t), u(t)\rangle\} d t,
$$

where $Q \in \mathcal{L}(X)$ and $Q_{1} \in \mathcal{L}(X)$ are self-adjoint but allowed to be indefinite; $R \in \mathcal{L}(U)$ and $R \gg 0$, i.e., there exists $\delta>0$ such that $\langle R u, u\rangle \geq \delta\|u\|_{U}^{2}$, $\forall u \in U$; and $y(\cdot)=y(\cdot ; x, u(\cdot))$ is given by (1.2). Hereafter, we call $Q$ and $Q_{1}$ the state weight operators of the cost functional. Usually, $Q_{1}$ or $Q$ is called a smoothing operator in the sense that, $\left(A^{*}\right)^{\beta} Q_{1} \in \mathcal{L}(X)$ or $\left(A^{*}\right)^{\beta} Q \in \mathcal{L}(X)$ for some nonnegative $\beta>\alpha-\frac{1}{2}$ respectively, (which is automatically satisfied with $\beta=0$ if $\left.0 \leq \alpha<\frac{1}{2}\right)$. In this paper, $Q_{1}$ and $Q$ are allowed to be not necessarily smoothing. Also, we point out that the state weight operators $Q$ and $Q_{1}$, and the operator $R$ are time-invariant only for the simplicity of presentation and they can be slightly relaxed. We need to specify the definition of $y(T)$ in $(1.5)$, since $\left.y(\cdot) \in L^{2}(0, T ; X)\right)$. Now, for any $u(\cdot) \in L^{2}(0, T ; U)$, we define

$$
\begin{aligned}
& \left\langle\int_{0}^{T} A^{\alpha} e^{-A(T-s)} B u(s) d s, z\right\rangle_{\left[D\left(A^{*}\right)\right]^{\prime}, D\left(A^{*}\right)} \\
& \triangleq\left\langle\int_{0}^{T} A^{\alpha-1} e^{-A(T-s)} B u(s) d s, A^{*} z\right\rangle_{X}, \quad \forall z \in D\left(A^{*}\right) .
\end{aligned}
$$

To this end, we point out that $\left[D\left(A^{*}\right)\right]^{\prime}$ coincides with the completion of $X$ under the norm $\|x\|_{-1}=\left\|A^{-1} x\right\|$. Hence, $\int_{0}^{T} A^{\alpha} e^{-A(T-s)} B u(s) d s \in$ $\left[D\left(A^{*}\right)\right]^{\prime}$ for all $u(\cdot) \in L^{2}(0, T ; U)$ and natural to define

$$
y(T)=e^{-A T} x+\int_{0}^{T} A^{\alpha} e^{-A(T-s)} B u(s) d s
$$

if $\int_{0}^{T} A^{\alpha} e^{-A(T-s)} B u(s) d s \in X \hookrightarrow\left[D\left(A^{*}\right)\right]^{\prime}$. This definition is the same as those in $[15,16]$. Define an operator $L_{T}: D\left(L_{T}\right) \subset L^{2}(0, T ; U) \mapsto X$ by

$$
L_{T} u=\int_{0}^{T} A^{\alpha} e^{-A(T-s)} B u(s) d s, \quad \forall u(\cdot) \in D\left(L_{T}\right),
$$


where $D\left(L_{T}\right)=\left\{u \in L^{2}(0, T ; U) \mid \int_{0}^{T} A^{\alpha} e^{-A(T-s)} B u(s) d s \in X\right\} . D\left(L_{T}\right)$ is dense in $L^{2}(0, T: U)$ since

$$
\left\{u(\cdot) \chi_{[0, T-\delta]}(\cdot)+u_{0} \chi_{[T-\delta, T]}(\cdot) \mid u(\cdot) \in \mathcal{U}[0, T], u_{0} \in U, \delta>0\right\} \subset D\left(L_{T}\right) .
$$

Our linear quadratic optimal control problem can be stated as follows:

LQ Problem. For given $x \in X$, find a $u(\cdot) \in D\left(L_{T}\right)$ so that the cost functional (1.5) is minimized.

In LQ differential game problems, the state weight operators of the cost functional appear to be indefinite in general. Molinari [19] pointed out that the state weight operators could be negative in certain LQ problems relating to network analysis. For the LQ problem with indefinite cost functional and with bounded control in Hilbert spaces, You [22] found a relation between the optimal feedback control and a Fredholm integral equation. A little later, Chen [4] directly discussed an equivalence between the Riccati equation (which is a nonlinear equation) and a linear Fredholm integral equation. See [20] for relevant results in an LQ differential game with multi-level of hierarchy. These results have also been further developed in [17]. Note that [17] studied the case with unbounded control and with the state weight operators being not necessarily smoothing, which includes the LQ problem for a parabolic equation with Neumann boundary control or pointwise control (for space dimension $n=1$ ).

In this paper, we focus on the LQ problem on a fixed time interval $[0, T], 0<T<+\infty$, with the state weight operators being indefinite and not necessarily smoothing, and with unbounded control including Dirichlet, Neumann boundary control and pointwise control (for space dimension $n \leq 3)$ for parabolic equations. We prove the equivalence between the solvability of the LQ problem and that of the corresponding two-point boundary value problem. It is shown that the solution to the two-point boundary value problem must be continuous, which leads to the continuity of the optimal control and the corresponding optimal state trajectory of the LQ problem. It is remarkable that this result here does not require any assumptions such as the uniqueness of the optimal control and the existence of the optimal control for each initial state in the space ( such a condition was traditionally assumed ahead in a similar content, see [15-17],etc.). We obtain the equivalence between the solvability of the two-point boundary value problem and that of a Fredholm integral equation, which yields a state feedback representation for the optimal control in terms of the solution to the Fredholm 
integral equation. Finally, we derive the closed-loop synthesis of the optimal control via the Riccati operator, a solution to the Riccati integral equation which exists under some mild conditions. In this procedure, we also obtain several regularity properties of the optimal control, the optimal state trajectory and the Riccati operator. Consequently, the Riccati operator obtained via the Riccati integral equation is also a solution to the Riccati differential equation in the weak sense. As an application, a simple explanatory example is given at the end. Our results extend those found in [17]. On the other hand, we note that, the LQ problems associated with parabolic type equations on the fixed time interval $[0, T], 0<T<+\infty$, with unbounded control studied in $[15,16]$ covered Dirichlet, Neumann boundary control and pointwise control (for space dimension $n \leq 3$ ) for parabolic equations; however the state weight operators of the cost functional were assumed to be nonnegative. Thus, our results also extend relevant results found in $[15,16]$.

2. Some preliminaries and notations. Note that in the case when $\alpha \geq \frac{1}{2}$, we do not know whether $A^{\alpha} e^{-A(T-\cdot)} B u(\cdot)$ is Bochner integrable in $X$ (equivalently, whether $\left\|A^{\alpha} e^{-A(T-\cdot)} B u(\cdot)\right\|$ is integrable) on the interval $[0, T]$ even if $u(\cdot) \in D\left(L_{T}\right)$. Therefore the following results is not obvious.

Proposition 2.1. If $u(\cdot) \in D\left(L_{T}\right)$, then it holds that

$$
\begin{aligned}
& \int_{0}^{T} e^{-A(T-s)} B u(s) d s \in D\left(A^{\alpha}\right), \\
& L_{T} u(\cdot)=A^{\alpha} \int_{0}^{T} e^{-A(T-s)} B u(s) d s .
\end{aligned}
$$

Conversely, if $u(\cdot) \in L^{2}(0, T ; U)$ satisfies $\int_{0}^{T} e^{-A(T-s)} B u(s) d s \in D\left(A^{\alpha}\right)$, then $u(\cdot) \in D\left(L_{T}\right)$.

Proof. For any $u(\cdot) \in D\left(L_{T}\right), A^{\alpha} e^{-A(T-\cdot)} B u(\cdot)$ is Bochner integrable on $[0, T]$ under the norm $\|\cdot\|_{-1}$ and the Bochner integral is $L_{T} u(\cdot)$. For $m=$ $1,2, \cdots$, define

$$
f_{m}(s)=A^{\alpha} e^{-A(T-s)} B u(s) \chi_{\left[0, T-\frac{T}{m}\right]}(s), \quad \forall 0 \leq s \leq T .
$$

By Lebesgue dominated convergence theorem, we have

$$
\int_{0}^{T} f_{m}(s) d s \stackrel{\|\cdot\|_{-1}}{\longrightarrow} \int_{0}^{T} A^{\alpha} e^{-A(T-s)} B u(s) d s \quad \text { as } m \rightarrow+\infty .
$$


Therefore, (1.1) and (2.3) imply that

$$
\begin{aligned}
0 & =\lim _{m \rightarrow+\infty}\left\|\int_{0}^{T} f_{m}(s) d s-L_{T} u(\cdot)\right\|_{-1} \\
& =\lim _{m \rightarrow+\infty}\left\|A^{-1+\alpha} \int_{0}^{T-\frac{1}{m} T} e^{-A(T-s)} B u(s) d s-A^{-1} L_{T} u(\cdot)\right\| .
\end{aligned}
$$

Since $A^{-1+\alpha} \in \mathcal{L}(X)$, it follows from (2.4) that

$$
A^{-1+\alpha} \int_{0}^{T} e^{-A(T-s)} B u(s) d s=A^{-1} L_{T} u(\cdot) .
$$

Due to $L_{T} u(\cdot) \in X$, we obtain $(2.1)$.

Conversely, if $A^{\alpha} \int_{0}^{T} e^{-A(T-s)} B u(s) d s \triangleq x(u(\cdot)) \in X$, then

$$
\int_{0}^{T} A^{-1+\alpha} e^{-A(T-s)} B u(s) d s=A^{-1+\alpha} \int_{0}^{T} e^{-A(T-s)} B u(s) d s=A^{-1} x(u(\cdot)) .
$$

On the other hand, $A^{\alpha} e^{-A(T-\cdot)} B u(\cdot)$ is Bochner integrable on $[0, T]$ under the norm $\|\cdot\|_{-1}$. Therefore there exists a sequence of simple functions $\phi_{k}:[0, T] \mapsto\left[D\left(A^{*}\right)\right]^{\prime}$ such that $\phi_{k}(\cdot) \stackrel{\text { a.e. }}{\longrightarrow} A^{\alpha} e^{-A(T-\cdot)} B u(\cdot)$ under the norm $\|\cdot\|_{-1}$ and

$$
\int_{0}^{T}\left\|A^{\alpha} e^{-A(T-s)} B u(s)-\phi_{k}(s)\right\|_{-1} d s \longrightarrow 0 \quad \text { as } k \rightarrow+\infty .
$$

Hence, we have

$$
\begin{aligned}
& \left\|x(u(\cdot))-\int_{0}^{T} \phi_{k}(s) d s\right\|_{-1}=\left\|A^{-1} x(u(\cdot))-A^{-1} \int_{0}^{T} \phi_{k}(s) d s\right\| \\
& =\left\|\int_{0}^{T} A^{-1+\alpha} e^{-A(T-s)} B u(s) d s-\int_{0}^{T} A^{-1} \phi_{k}(s) d s\right\| \\
& \leq \int_{0}^{T}\left\|A^{\alpha} e^{-A(T-s)} B u(s)-\phi_{k}(s)\right\|_{-1} d s \rightarrow 0, \quad \text { as } k \rightarrow+\infty
\end{aligned}
$$

which means the Bochner integral of $A^{\alpha} e^{-A(T-\cdot)} B u(\cdot)$ on $[0, T]$ under the norm $\|\cdot\|_{-1}$ is $x(u(\cdot))$. The proof is completed. 
It follows from Proposition 2.1 that $L_{T}$ is closed because of the closedness of $A^{\alpha}$.

In the sequel of this paper, we will use the following notations: For any given $t \in[0, T)$, we also consider the following state equation

$$
y_{t, x}(s)=e^{-A(s-t)} x+\int_{t}^{s} A^{\alpha} e^{-A(s-r)} B u(r) d r, \quad s \in[t, T], \quad(0 \leq \alpha<1),
$$

and the corresponding cost functional

$$
\begin{aligned}
J_{t, x}(u(\cdot))= & \left\langle Q_{1} y_{t, x}(T), y_{t, x}(T)\right\rangle \\
& +\int_{t}^{T}\left\{\left\langle Q y_{t, x}(s), y_{t, x}(s)\right\rangle+\langle R u(s), u(s)\rangle\right\} d s .
\end{aligned}
$$

Denote $\mathcal{X}[t, T]=L^{2}(t, T ; X)$ and $\mathcal{U}[t, T]=L^{2}(t, T ; U)$ and define $L(t), L_{T}(t)$ as follows:

$$
\left\{\begin{array}{l}
(L(t) u)(s)=\int_{t}^{s} A^{\alpha} e^{-A(s-r)} B u(r) d r, \quad \text { for } s \in[t, T], \quad u(\cdot) \in \mathcal{U}[t, T] \\
L_{T}(t) u=\int_{t}^{T} A^{\alpha} e^{-A(T-r)} B u(r) d r, \quad \text { for } \quad u(\cdot) \in D\left(L_{T}(t)\right) ; \quad(2.10) \\
D\left(L_{T}(t)\right)=\left\{u(\cdot) \in \mathcal{U}[t, T] \mid \int_{t}^{T} A^{\alpha} e^{-A(T-r)} B u(r) d r \in X\right\} .
\end{array}\right.
$$

Here $L_{T}(0)=L_{T}$ and $D\left(L_{T}(t)\right)$ is also dense in $\mathcal{U}[t, T]$. Similar to Proposition 2.1, we have:

Proposition 2.2. If $u(\cdot) \in D\left(L_{T}(t)\right)$, then it holds that

$$
\begin{aligned}
& \int_{t}^{T} e^{-A(T-s)} B u(s) d s \in D\left(A^{\alpha}\right), \\
& L_{T}(t) u(\cdot)=A^{\alpha} \int_{t}^{T} e^{-A(T-s)} B u(s) d s .
\end{aligned}
$$

Conversely, if $u(\cdot) \in \mathcal{U}[t, T]$ satisfies $\int_{t}^{T} e^{-A(T-s)} B u(s) d s \in D\left(A^{\alpha}\right)$, then $u(\cdot) \in D\left(L_{T}(t)\right)$.

Therefore, $L_{T}(t)$ is closed. By Lemma 1.1 in [17, p.363],

$$
L(t) \in \mathcal{L}(\mathcal{U}[t, T] ; \mathcal{X}[t, T]) .
$$


The adjoint operators $L^{*}(t)$ and $L_{T}^{*}(t)$ of $L(t)$ and $L_{T}(t)$ respectively are given by

$$
\left\{\begin{array}{l}
{\left[L^{*}(t) y\right](s)=\int_{s}^{T} B^{*}\left(A^{*}\right)^{\alpha} e^{-A^{*}(\sigma-s)} y(\sigma) d \sigma, \text { for } s \in[t, T], y \in \mathcal{X}[t, T]} \\
{\left[L_{T}^{*}(t) x\right](s)=B^{*}\left(A^{*}\right)^{\alpha} e^{-A^{*}(T-s)} x, \quad \text { for } \quad s \in[t, T], \quad x \in D\left(L_{T}^{*}(t)\right) .}
\end{array}\right.
$$

Here, $L^{*}(t) \in \mathcal{L}(\mathcal{X}[t, T] ; \mathcal{U}[t, T])$, and $\overline{D\left(L_{T}^{*}(t)\right)}=X$ because $D\left(\left(A^{*}\right)^{\alpha}\right) \subset$ $D\left(L_{T}^{*}(t)\right)$ and $\overline{D\left(\left(A^{*}\right)^{\alpha}\right)}=X$. It follows from $\overline{D\left(L_{T}(t)\right)}=X$ that $L_{T}^{*}(t)$ : $D\left(L_{T}^{*}(t)\right) \subseteq X \mapsto \mathcal{U}[t, T]$ is closed (see $\left.[12,15-17]\right)$. In what follows, we let

$$
\left\{\begin{array}{l}
\Phi_{0}(t)=R+L^{*}(t) Q L(t), \quad \Theta_{0}(t)=L^{*}(t) Q e^{-A(\cdot-t)} \\
\Gamma(t)=\int_{t}^{T} e^{-A^{*} s} Q e^{-A s} d s+e^{-A^{*}(T-t)} Q_{1} e^{-A(T-t)}
\end{array}\right.
$$

For any given $t \in[0, T)$, we pose the following problem:

Problem $(\mathbf{L Q})_{t}$. For given $x \in X$, find a $u(\cdot) \in D\left(L_{T}(t)\right)$ so that the cost functional (2.9) is minimized.

If there exists a $\bar{u}_{t, x}(\cdot) \in D\left(L_{T}(t)\right)$ such that

$$
J_{t, x}\left(\bar{u}_{t, x}(\cdot)\right)=\inf _{u(\cdot) \in D\left(L_{T}(t)\right)} J_{t, x}(u(\cdot))>-\infty,
$$

we say that Problem $(L Q)_{t}$ is solvable at $x \in X$. In this case, we call the control $\bar{u}_{t, x}(\cdot)$ an optimal control of Problem $(L Q)_{t}$, the corresponding trajectory $\bar{y}_{t, x}(\cdot)$ and the pair $\left(\bar{u}_{t, x}(\cdot), \bar{y}_{t, x}(\cdot)\right)$ are called an optimal trajectory and an optimal pair, respectively.

Remark 2.3. In the LQ problem of (1.2) with the cost functional (1.5) on a fixed time interval $[0, T], 0<T<+\infty$, a main difficulty arises from that the pointwise value $y(T ; x, u(\cdot))$ appears in the cost functional (1.5) but the state trajectory $y(\cdot: x, u(\cdot))$ is only in $L^{2}(0, T ; X)$. Therefore, we must deal with an unbounded operator $u(\cdot) \mapsto y(T ; x, u(\cdot))$ on some dense set in $L^{2}(0, T ; U)$. In an ordinary way, it is reasonable to define a pointwise value only for functions in $C([0, T] ; X)$. Thus, a notion of an operator $L_{0}$ was proposed in [17]:

$$
\left\{\begin{array}{l}
D\left(L_{0}\right) \triangleq\left\{u(\cdot) \in L^{2}(0, T ; U) \mid \lim _{t \rightarrow T} \int_{0}^{t} A^{\alpha} e^{-A(t-s)} B u(s) d s \text { exists }\right\} \\
L_{0} u(\cdot)=\lim _{t \rightarrow T} \int_{0}^{t} A^{\alpha} e^{-A(t-s)} B u(s) d s, \quad \forall u(\cdot) \in D\left(L_{0}\right) .
\end{array}\right.
$$


For convenience, [17] discussed the LQ problem of (1.2) with (1.5) in the case $y(T ; x, u(\cdot))=e^{-A T} x+L_{1} u(\cdot)$ on $D\left(L_{1}\right) \subset L^{2}(0, T ; U)$ where $L_{1}$ is a closed extension of $L_{0}$ and defined directly by

$$
\left\{\begin{array}{l}
D\left(L_{1}\right) \triangleq\left\{u(\cdot) \in L^{2}(0, T ; U) \mid \int_{0}^{T} e^{-A(T-s)} B u(s) d s \in D\left(A^{\alpha}\right)\right\} \\
L_{1} u(\cdot)=A^{\alpha} \int_{0}^{T} e^{-A(T-s)} B u(s) d s, \quad \forall u(\cdot) \in D\left(L_{1}\right) .
\end{array}\right.
$$

Proposition 2.1 tells us that $L_{T}=L_{1}$. Therefore, [17] and [15,16] gave the same definition of $y(T ; x, u(\cdot))$ in two different approaches. In this paper, we focus on the LQ problem of (1.2) with (1.5) on $D\left(L_{T}\right)$ in this case $y(T ; x, u(\cdot))=e^{-A T} x+L_{T} u(\cdot)$. However, all the conclusions in this paper can be established similarly to another case with $y(T ; x, u(\cdot))=e^{-A T} x+$ $L_{0} u(\cdot)$ on $D\left(L_{0}\right)$.

3. Some inequalities. In this section, we establish some inequalities, which can be applied to estimate solutions to the two-point boundary value problem in various norms.

Lemma 3.1. Let $0 \leq \lambda, \mu<1 ; C_{1}, C_{2}>0$ and $-\infty<a<b<+\infty$. Let $K(\cdot, \cdot)$ and $L(\cdot, \cdot)$ be Lebesgue measurable on $[a, b] \times[a, b]$ with

$$
0 \leq K(\xi, \zeta) \leq \frac{C_{1}}{|\xi-\zeta|^{\lambda}}, \quad 0 \leq L(\xi, \zeta) \leq \frac{C_{2}}{|\xi-\zeta|^{\mu}}, \quad \text { for } a \leq \xi, \zeta \leq b
$$

and

$$
M(\xi, \zeta) \triangleq \int_{a}^{b} K(\xi, \eta) L(\eta, \zeta) d \eta
$$

Then there exists a $C>0$, such that for all $(\xi, \zeta) \in[a, b] \times[a, b]$

$$
M(\xi, \zeta) \leq \begin{cases}C, & \text { if } \lambda+\mu<1 \\ \frac{C}{|\xi-\zeta|^{\lambda+\mu-1}}, & \text { if } \lambda+\mu>1\end{cases}
$$

Proof. This lemma was proved in [11, p.14].

Our main result of this section is the following: 
Theorem 3.2. Let $0 \leq \lambda, \mu<1, C_{1}, C_{2}, C_{3}>0$, and $-\infty<a<b<+\infty$. For any given $t \in[a, b)$, suppose that $u(\cdot) \in L^{1}(t, b)$ satisfies

$$
0 \leq u(s) \leq C_{1}+\frac{C_{2}}{|b-s|^{\mu}}+C_{3} \int_{t}^{b} \frac{u(\tau)}{|s-\tau|^{\lambda}} d \tau, \quad \text { for } \quad t \leq s<b
$$

Then it holds that

$$
\begin{gathered}
u(s) \leq K\left(C_{1}+\frac{C_{2}}{|b-s|^{\mu}}+C_{3} \int_{t}^{b} u(\tau) d \tau\right), \quad \text { for } \quad t \leq s<b, \\
\int_{t}^{b} \frac{u(\tau)}{|s-\tau|^{\gamma}} d \tau \leq K_{\gamma}\left(C_{1}+\frac{C_{2}}{|b-s|^{\mu}}+C_{3} \int_{t}^{b} u(\tau) d \tau\right), \quad \text { for } t \leq s<b,
\end{gathered}
$$

where $\gamma \in[0,1)$, and $K, K_{\gamma}>0$ are constants independent of $t \in[a, b)$ but $K_{\gamma}$ is dependent of $\gamma$.

Proof. For $t \in[a, b)$ given, we denote

$$
\left\{\begin{array}{l}
f(s) \triangleq C_{1}+\frac{C_{2}}{|b-s|^{\mu}}, \quad \text { for } \quad t \leq s<b \\
K(s, \tau) \triangleq \frac{1}{|s-\tau|^{\lambda}}, \quad \text { for } \quad t \leq s, \tau \leq b
\end{array}\right.
$$

and for $v \in L^{1}(t, b)$ nonnegative, define

$$
[S v](s)=C_{3} \int_{t}^{b} \frac{v(\tau)}{|s-\tau|^{\lambda}} d \tau, \quad \text { for } \quad t \leq s<b
$$

We have the following estimates:

$$
\begin{aligned}
& \int_{t}^{b} \frac{1}{|s-\tau|^{\lambda}|b-\tau|^{\mu}} d \tau=\int_{t}^{s} \frac{1}{|s-\tau|^{\lambda}|b-\tau|^{\mu}} d \tau+\int_{s}^{b} \frac{1}{|\tau-s|^{\lambda}|b-\tau|^{\mu}} d \tau \\
& \leq \frac{1}{|b-s|^{\mu}} \int_{t}^{s} \frac{1}{|s-\tau|^{\lambda}} d \tau+B(1-\lambda, 1-\beta) \frac{1}{|b-s|^{\lambda+\mu-1}} \\
& \leq(b-a)^{1-\lambda} \max \left\{\frac{1}{1-\lambda}, B(1-\lambda, 1-\beta)\right\} \frac{1}{|b-s|^{\mu}},
\end{aligned}
$$


where

$$
B(1-\lambda, 1-\beta)=\int_{0}^{1} \theta^{-\lambda}(1-\theta)^{-\beta} d \theta
$$

is the Beta function. Let

$$
C_{4}=(b-a)^{1-\lambda} \max \left\{\frac{1}{1-\lambda}, B(1-\lambda, 1-\beta)\right\} .
$$

We obtain from (3.8) that

$$
\begin{aligned}
{[S f(\cdot)](s) } & =C_{1} C_{3} \int_{t}^{b} \frac{1}{|s-\tau|^{\lambda}} d \tau+C_{2} C_{3} \int_{t}^{b} \frac{1}{|s-\tau|^{\lambda}|b-\tau|^{\mu}} d \tau \\
& \leq C_{3} C_{4} f(s), \quad \text { for } t \leq s<b .
\end{aligned}
$$

Denote $n=\left[\frac{1}{1-\lambda}\right]+1$, it follows from (3.3) that

$$
\begin{aligned}
0 \leq u(s) & \leq f(s)+[S u(\cdot)](s) \leq f(s)+[S f(\cdot)](s)+\left[S^{2} u(\cdot)\right](s) \\
& \leq \sum_{k=0}^{n-1}\left[S^{k} f(\cdot)\right](s)+\left[S^{n} u(\cdot)\right](s) .
\end{aligned}
$$

By Fubini Theorem and Lemma 3.1, we obtain that there exists a $C_{5}>0$ independent of $t \in[a, b)$ such that

$$
\left[S^{n} u(\cdot)\right](s)=C_{3}^{n} \int_{t}^{b} K_{n}(s, \tau) u(\tau) d \tau \leq C_{5} C_{3} \int_{t}^{b} u(\tau) d \tau
$$

where $K_{n}(\cdot, \cdot)$ is the n-time iterated kernel of the kernel $K(\cdot, \cdot)$ defined by:

$$
\left\{\begin{array}{l}
K_{1}(\xi, \zeta) \triangleq K(\xi, \zeta), \\
K_{m}(\xi, \zeta) \triangleq \int_{t}^{b} K_{m-1}(\xi, \eta) K(\eta, \zeta) d \eta,
\end{array} \text { for } t \leq \xi, \zeta<b, m \geq 2 .\right.
$$

Combining (3.9), (3.10) and (3.11), we see that there exists a $K>0$ independent of $t \in[a, b)$ such that

$$
u(s) \leq K\left(C_{1}+\frac{C_{2}}{|b-s|^{\mu}}+C_{3} \int_{t}^{b} u(\tau) d \tau\right), \quad \text { for } \quad t \leq s<b
$$


and, for $t \leq s<b$,

$$
\begin{aligned}
\int_{t}^{b} \frac{u(\tau)}{|s-\tau|^{\lambda}} d \tau & =\frac{[S u(\cdot)](s)}{C_{3}} \leq \frac{1}{C_{3}}\left\{\sum_{k=1}^{n}\left[S^{k} f(\cdot)\right](s)+\left[S^{n+1} u(\cdot)\right](s)\right\} \\
& \leq K\left(C_{1}+\frac{C_{2}}{|b-s|^{\mu}}+C_{3} \int_{t}^{b} u(\tau) d \tau\right)
\end{aligned}
$$

If $0 \leq \gamma \leq \lambda$, then

$$
\begin{aligned}
\int_{t}^{b} \frac{u(\tau)}{|s-\tau|^{\gamma}} d \tau & =\int_{t}^{b}|s-\tau|^{\lambda-\gamma} \frac{u(\tau)}{|s-\tau|^{\lambda}} d \tau \\
& \leq(b-a)^{\lambda-\gamma} \int_{t}^{b} \frac{u(\tau)}{|s-\tau|^{\lambda}} d \tau, \quad \text { for } \quad t \leq s<b
\end{aligned}
$$

Estimates (3.14) and (3.15) imply that there exists a $K_{\gamma} \triangleq K(b-a)^{\lambda-\gamma}$ independent of $t \in[a, b)$ such that (3.5) hold. If $\lambda<\gamma<1$, then it follows from (3.3) that

$$
0 \leq u(s) \leq C_{1}+\frac{C_{2}}{|b-s|^{\mu}}+C_{3}^{\prime} \int_{t}^{b} \frac{u(\tau)}{|s-\tau|^{\gamma}} d \tau,
$$

for $t \leq s<b$, where $C_{3}^{\prime}=C_{3}(b-a)^{\gamma-\lambda}$. Applying (3.14) to (3.16), we obtain that there exists a $K_{\gamma}>0$ independent of $t \in[a, b)$ such that (3.5) hold.

4. The LQ problem and the two-point boundary value problem. In this section, we prove the equivalence between the solvability of Problem $(L Q)_{t}$ and the solvability of the corresponding two-point boundary value problem in Theorem 4.2. Our result is a modification of a result found in [17, pp.381 and 386]. In general, the state trajectory of the control system (1.2) is not necessarily continuous if $\alpha \geq \frac{1}{2}$. Applying proper inequalities from Theorem 3.2, we can get the estimates for the solution to the two-point boundary value problem and prove that the solution must be continuous, namely the optimal control and the optimal trajectory must be continuous, without additional assumptions on $Q$ and $Q_{1}$ like in [15-17]. Note that in Theorem 4.5 we do not require the uniqueness of the optimal control at $x \in X$ or the solvability of Problem $(L Q)_{t}$ at other $x \in X$.

The following theorem gives a necessary and sufficient condition for Problem $(L Q)_{t}$ to be solvable at $x \in X$. 
Theorem 4.1. For given $t \in[0, T), \bar{u}_{t, x}(\cdot) \in D\left(L_{T}(t)\right)$ is an optimal control for Problem $(L Q)_{t}$ at $x \in X$ if and only if

$$
L_{T}^{*}(t) Q_{1}\left[L_{T}(t) \bar{u}_{t, x}(\cdot)+e^{-A(T-t)} x\right]+\Phi_{0}(t) \bar{u}_{t, x}(\cdot)+\Theta_{0}(t) x=0 ;
$$

and

$$
\left\langle\Phi_{0}(t) u(\cdot), u(\cdot)\right\rangle+\left\langle Q_{1} L_{T}(t) u(\cdot), L_{T}(t) u(\cdot)\right\rangle \geq 0, \quad \forall u(\cdot) \in D\left(L_{T}(t)\right) .
$$

Proof. For $u(\cdot) \in D\left(L_{T}(t)\right)$, it is holds that

$$
\begin{aligned}
y_{t, x}(s)= & e^{-A(s-t)} x+[L(t) u(\cdot)](s), \quad y_{t, x}(T)=e^{-A(T-t)} x+L_{T}(t) u(\cdot) . \\
J_{t, x}(u(\cdot)) & =\left\langle\Phi_{0}(t) u(\cdot), u(\cdot)\right\rangle+\left\langle Q_{1} L_{T}(t) u(\cdot), L_{T}(t) u(\cdot)\right\rangle \\
& +2\left\{\left\langle\Theta_{0}(t) x, u(\cdot)\right\rangle+\left\langle Q_{1} e^{-A(T-t)}, L_{T}(t) u(\cdot)\right\rangle\right\}+\langle\Gamma(t) x, x\rangle .
\end{aligned}
$$

Therefore, Proposition 2.1 in [17, p.371], Theorem 2.2 in [17, p.372] and Proposition 2.2 lead to our conclusion immediately.

Theorem 4.2. For given $t \in[0, T)$, let (4.2) hold. Then Problem $(L Q)_{t}$ is solvable at $x \in X$ if and only if there exists a pair $\left(y_{t, x}(\cdot), \psi_{t, x}(\cdot)\right) \in$ $\mathcal{X}[t, T] \times L^{1}(t, T ; X)$ with

$$
B^{*} \psi_{t, x}(\cdot) \in \mathcal{X}[t, T], \quad \int_{t}^{T} A^{\alpha} e^{-A(T-r)} B R^{-1} B^{*} \psi_{t, x}(r) d r \in X,
$$

solving the following two-point boundary value problem

$$
\left\{\begin{array}{cl}
y_{t, x}(s)=e^{-A(s-t)} x+\int_{t}^{s} A^{\alpha} e^{-A(s-r)} B R^{-1} B^{*} \psi_{t, x}(r) d r, & s \in[t, T], \\
\psi_{t, x}(s)=-\left(A^{*}\right)^{\alpha} e^{-A^{*}(T-s)} Q_{1} y_{t, x}(T) & \\
-\int_{s}^{T}\left(A^{*}\right)^{\alpha} e^{-A^{*}(\sigma-s)} Q y_{t, x}(\sigma) d \sigma, & s \in[t, T] .
\end{array}\right.
$$

In this case

$$
\bar{u}_{t, x}(s)=R^{-1} B^{*} \psi_{t, x}(s), \quad s \in[t, T]
$$

gives an optimal control and $y_{t, x}(\cdot)$ is the corresponding optimal trajectory.

Proof. From Theorem 4.1, we know that under (4.2), Problem $(L Q)_{t}$ is solvable at $x \in X$ with an optimal control $\bar{u}_{t, x}(\cdot)$ if and only if (4.1) holds. 
If $\bar{u}_{t, x}(\cdot)$ is an optimal control of Problem $(L Q)_{t}$ at $x \in X$, then $\bar{y}_{t, x}(\cdot)=$ $e^{-A(\cdot-t)} x+\left[L(t) \bar{u}_{t, x}(\cdot)\right](\cdot) \in \mathcal{X}[t, T]$ is the corresponding optimal state trajectory. Since $\bar{u}_{t, x}(\cdot) \in D\left(L_{T}(t)\right), \bar{y}_{t, x}(T)=e^{-A(T-t)} x+L_{T}(t) \bar{u}_{t, x}(\cdot)$ is well defined. Set

$$
\begin{aligned}
\psi_{t, x}(s)= & -\left(A^{*}\right)^{\alpha} e^{-A^{*}(T-s)} Q_{1} \bar{y}_{t, x}(T) \\
& -\int_{s}^{T}\left(A^{*}\right)^{\alpha} e^{-A^{*}(\sigma-s)} Q \bar{y}_{t, x}(\sigma) d \sigma, \quad s \in[t, T] .
\end{aligned}
$$

By (1.1), the first term in the right-hand side of (4.8) is in $L^{1}(t, T ; X)$, and the second term is in $\mathcal{X}[t, T]$ by a similar proof of Lemma 1.1 in $[17$, p.363]. Hence $\psi_{t, x}(\cdot)$ defined by $(4.8)$ is in $L^{1}(t, T ; X)$. Next, by $(4.1),(2.12)$ and (2.13), we have, from (4.8), that

$$
\begin{aligned}
& B^{*} \psi_{t, x}(\cdot)=-L_{T}^{*}(t) Q_{1} \bar{y}_{t, x}(T)-L^{*}(t) Q \bar{y}_{t, x}(\cdot) \\
& =-L_{T}^{*}(t) Q_{1}\left[L_{T}(t) \bar{u}_{t, x}(\cdot)+e^{-A(T-t)} x\right]-L^{*}(t) Q e^{-A(\cdot-t)} x \\
& \quad-L^{*}(t) Q L(t) \bar{u}_{t, x}(\cdot)=R \bar{u}_{t, x}(\cdot) .
\end{aligned}
$$

This yields (4.7). Hence, (4.5) holds for $\psi_{t, x}(\cdot)$. Clearly, $\left(\bar{y}_{t, x}(\cdot), \psi_{t, x}(\cdot)\right) \in$ $\mathcal{X}[t, T] \times L^{1}(t, T ; X)$ satisfies $(4.6)$ a.e. on $[t, T]$.

Conversely, let $\left(\bar{y}_{t, x}(\cdot), \psi_{t, x}(\cdot)\right) \in \mathcal{X}[t, T] \times L^{1}(t, T ; X)$ be a solution to (4.6) with the property (4.5). Then we define $\bar{u}_{t, x}(\cdot)$ by (4.7). Clearly (4.5) implies $\bar{u}_{t, x}(\cdot) \in D\left(L_{T}(t)\right)$. It follows from (4.6), (4.7) and (2.12) that $\bar{y}_{t, x}(\cdot)$ is the state trajectory of $(1.2)$ with control $\bar{u}_{t, x}(\cdot)$ and

$$
\begin{aligned}
R \bar{u}_{t, x}(\cdot) & =B^{*} \psi_{t, x}(\cdot)=-L_{T}^{*}(t) Q_{1} \bar{y}_{t, x}(T)-L^{*}(t) Q \bar{y}_{t, x}(\cdot) \\
& =-L_{T}^{*}(t) Q_{1}\left[L_{T}(t) \bar{u}_{t, x}(\cdot)+e^{-A(T-t)} x\right] \\
& -L^{*}(t) Q e^{-A(\cdot-t)} x-L^{*}(t) Q L(t) \bar{u}_{t, x}(\cdot) .
\end{aligned}
$$

Hence $\bar{u}_{t, x}(\cdot) \in D\left(L_{T}(t)\right)$ satisfies (4.1).

Definition 4.3. For given $t \in[0, T),\left(y_{t, x}(\cdot), \psi_{t, x}(\cdot)\right) \in L^{1}(t, T ; X) \times$ $L^{1}(t, T ; X)$ is called a solution to $(4.6)$ if $\left(y_{t, x}(\cdot), \psi_{t, x}(\cdot)\right)$ satisfies (4.6) almost everywhere on $[t, T]$ and $y_{t, x}(T)$ is well-defined, i.e.,

$$
\int_{t}^{T} A^{\alpha} e^{-A(T-\tau)} B R^{-1} B^{*} \psi_{t, x}(\tau) d \tau \in X
$$


Theorem 4.4. For given $t \in[0, T)$, suppose that

$$
\left(y_{t, x}(\cdot), \psi_{t, x}(\cdot)\right) \in L^{1}(t, T ; X) \times L^{1}(t, T ; X)
$$

is a solution to (4.6). Then

$$
\begin{aligned}
& \left\{\begin{array}{l}
\left\|y_{t, x}(s)\right\| \leq K\left(\|x\|+\frac{\left\|Q_{1} y_{t, x}(T)\right\|}{|T-s|^{\alpha}}+\int_{t}^{T}\left\|y_{t, x}(\tau)\right\| d \tau\right), \\
\int_{t}^{T} \frac{\left\|y_{t, x}(\tau)\right\|}{|s-\tau|^{\gamma}} d \tau \leq K_{\gamma}\left(\|x\|+\frac{\left\|Q_{1} y_{t, x}(T)\right\|}{|T-s|^{\alpha}}+\int_{t}^{T}\left\|y_{t, x}(\tau)\right\| d \tau\right) ;
\end{array}\right. \\
& \left\{\begin{array}{l}
\left\|\psi_{t, x}(s)\right\| \leq K\left(\|x\|+\frac{\left\|Q_{1} y_{t, x}(T)\right\|}{|T-s|^{\alpha}}+\int_{t}^{T}\left\|B^{*} \psi_{t, x}(\tau)\right\| d \tau\right), \\
\int_{t}^{T} \frac{\left\|\psi_{t, x}(\tau)\right\|}{|s-\tau|^{\gamma}} d \tau \leq K_{\gamma}\left(\|x\|+\frac{\left\|Q_{1} y_{t, x}(T)\right\|}{|T-s|^{\alpha}}+\int_{t}^{T}\left\|B^{*} \psi_{t, x}(\tau)\right\| d \tau\right) ;
\end{array}\right. \\
& \left\{\begin{array}{c}
\left\|\left(A^{*}\right)^{\theta-\alpha} \psi_{t, x}(s)\right\| \leq K(\theta)\left(\|x\|+\frac{\left\|Q_{1} y_{t, x}(T)\right\|}{|T-s|^{\theta}}+\int_{t}^{T}\left\|y_{t, x}(\sigma)\right\| d \sigma\right), \\
\int_{t}^{T} \frac{\left\|\left(A^{*}\right)^{\theta-\alpha} \psi_{t, x}(\tau)\right\|}{|s-\tau|^{\gamma}} d \tau \leq K_{\gamma}(\theta)\left(\|x\|+\frac{\left\|Q_{1} y_{t, x}(T)\right\|}{|T-s|^{\theta}}\right. \\
\left.+\int_{t}^{T}\left\|y_{t, x}(\sigma)\right\| d \sigma\right)
\end{array}\right.
\end{aligned}
$$

for $t \leq s<T$, where $\gamma \in[0,1), \theta \in[0,1)$, and $K, K_{\gamma}, K(\theta), K_{\gamma}(\theta)>0$ are constants independent of $t \in[0, T)$ and $\left(y_{t, x}(\cdot), \psi_{t, x}(\cdot)\right)$, but $K(\theta), K_{\gamma}(\theta)$ dependent of $\theta$ and $K_{\gamma}, K_{\gamma}(\theta)$ dependent of $\gamma$.

Proof. By (1.1) and $B^{*} \in \mathcal{L}(X, U)$, it follows from (4.6) that for some $C>0$ independent of $t \in[0, T)$

$$
\left\{\begin{array}{l}
\left\|y_{t, x}(s)\right\| \leq C\left(\|x\|+\int_{t}^{s} \frac{\left\|B^{*} \psi_{t, x}(\tau)\right\|}{|s-\tau|^{\alpha}} d \tau\right), \\
\left\|B^{*} \psi_{t, x}(s)\right\| \leq C\left(\frac{\left\|Q_{1} y_{t, x}(T)\right\|}{|T-s|^{\alpha}}+\int_{s}^{T} \frac{\left\|y_{t, x}(\sigma)\right\|}{|\sigma-s|^{\alpha}} d \sigma\right),
\end{array} \quad \text { for } t \leq s<T .\right.
$$


A straightforward calculation easily shows that,

$$
\begin{aligned}
& \int_{t}^{s} \int_{\tau}^{T} \frac{\left\|y_{t, x}(\sigma)\right\|}{|s-\tau|^{\alpha}|\sigma-\tau|^{\alpha}} d \sigma d \tau \\
& =\int_{t}^{s} \int_{\tau}^{s} \frac{\left\|y_{t, x}(\sigma)\right\|}{|s-\tau|^{\alpha}|\sigma-\tau|^{\alpha}} d \sigma d \tau+\int_{t}^{s} \int_{s}^{T} \frac{\left\|y_{t, x}(\sigma)\right\|}{|s-\tau|^{\alpha}|\sigma-\tau|^{\alpha}} d \sigma d \tau \\
& \leq \int_{t}^{s} \int_{t}^{\sigma} \frac{1}{|\sigma-\tau|^{\alpha}} d \tau \frac{\left\|y_{t, x}(\sigma)\right\|}{|s-\sigma|^{\alpha}} d \sigma+\int_{s}^{T} \int_{t}^{s} \frac{1}{|s-\tau|^{\alpha}} d \tau \frac{\left\|y_{t, x}(\sigma)\right\|}{|\sigma-s|^{\alpha}} d \sigma \\
& \leq \frac{T^{1-\alpha}}{1-\alpha} \int_{t}^{T} \frac{\left\|y_{t, x}(\sigma)\right\|}{|s-\sigma|^{\alpha}} d \sigma, \quad \text { for } t \leq s<T .
\end{aligned}
$$

Consequently, (4.13) and the above imply

$$
\begin{aligned}
\left\|y_{t, x}(s)\right\| \leq & C\left\{\|x\|+\int_{t}^{s} \frac{C}{|s-\tau|^{\alpha}}\left[\frac{\left\|Q_{1} y_{t, x}(T)\right\|}{|T-\tau|^{\alpha}}+\int_{\tau}^{T} \frac{\left\|y_{t, x}(\sigma)\right\|}{|\sigma-\tau|^{\alpha}} d \sigma\right] d \tau\right\} \\
\leq & C\left[\|x\|+\frac{\left\|Q_{1} y_{t, x}(T)\right\|}{|T-s|^{\alpha}} \int_{t}^{s} \frac{C}{|s-\tau|^{\alpha}} d \tau\right. \\
& \left.+\int_{t}^{s} \int_{\tau}^{T} \frac{C\left\|y_{t, x}(\sigma)\right\|}{|s-\tau|^{\alpha}|\sigma-\tau|^{\alpha}} d \sigma d \tau\right] \\
\leq & C_{1}\left[\|x\|+\frac{\left\|Q_{1} y_{t, x}(T)\right\|}{|T-s|^{\alpha}}+\int_{t}^{T} \frac{\left\|y_{t, x}(\sigma)\right\|}{|s-\sigma|^{\alpha}} d \sigma\right]
\end{aligned}
$$

where $C_{1}=C \max \left\{1, \frac{C T^{1-\alpha}}{1-\alpha}\right\}$. Similarly, it holds that for some $C_{2}>0$ independent of $t \in[0, T)$

$$
\left\|B^{*} \psi_{t, x}(s)\right\| \leq C_{2}\left[\|x\|+\frac{\left\|Q_{1} y_{t, x}(T)\right\|}{|T-s|^{\alpha}}+\int_{t}^{T} \frac{\left\|B^{*} \psi_{t, x}(\tau)\right\|}{|s-\tau|^{\alpha}} d \tau\right]
$$

for $t \leq s<T$. By Theorem 3.2 and $B^{*} \in \mathcal{L}(X, U)$, we obtain (4.10) and (4.11), from (4.14) and (4.15) respectively. From (4.6) and (1.1), we also have

$$
\left\{\begin{array}{l}
\left\|y_{t, x}(s)\right\| \leq C_{3}\left(\|x\|+\int_{t}^{s} \frac{\left\|\left(A^{*}\right)^{\theta-\alpha} \psi_{t, x}(\tau)\right\|}{|s-\tau|^{\alpha}} d \tau\right) \\
\left\|\left(A^{*}\right)^{\theta-\alpha} \psi_{t, x}(s)\right\| \leq C_{3}\left(\frac{\left\|Q_{1} y_{t, x}(T)\right\|}{|T-s|^{\theta}}+\int_{s}^{T} \frac{\left\|y_{t, x}(\sigma)\right\|}{|\sigma-s|^{\theta}} d \sigma\right),
\end{array}\right.
$$


for $t \leq s<T$, where $C_{3}>0$ is a constant independent of $t \in[0, T)$ but dependent of $\theta \in[0,1)$. By the second equality of (4.6) and Fubini theorem, we have

$$
\int_{t}^{T}\left\|\left(A^{*}\right)^{\theta-\alpha} \psi_{t, x}(\tau)\right\| d \tau \leq C_{4}\left(\left\|Q_{1} y_{t, x}(T)\right\|+\int_{t}^{T}\left\|y_{t, x}(\sigma)\right\| d \sigma\right)
$$

where $C_{4}=\frac{T^{1-\theta}}{1-\theta} C_{3}$. Similar to (4.15), we can derive from (4.16) through a straightforward calculation that

$$
\left\|\left(A^{*}\right)^{\theta-\alpha} \psi_{t, x}(s)\right\| \leq C_{5}\left(\|x\|+\frac{\left\|Q_{1} y_{t, x}(T)\right\|}{|T-s|^{\theta}}+\int_{s}^{T} \frac{\left\|\left(A^{*}\right)^{\theta-\alpha} \psi_{t, x}(\tau)\right\|}{|\tau-s|^{\theta}} d \tau\right),
$$

where $C_{5}$ is also a constant independent of $t \in[0, T)$ but dependent of $\theta \in[0,1)$. By (4.17) and (4.18), (4.12) can be derived similarly.

Theorem 4.5. For given $t \in[0, T)$, suppose that

$$
\left(y_{t, x}(\cdot), \psi_{t, x}(\cdot)\right) \in L^{1}(t, T ; X) \times L^{1}(t, T ; X)
$$

is a solution to (4.6). Then both $y_{t, x}(\cdot)$ and $\left(A^{*}\right)^{\beta} \psi_{t, x}(\cdot)$ are continuous on $[t, T)$, and $\left(A^{*}\right)^{\beta} y_{t, x}(\cdot)$ is continuous on $(t, T)$ for any $\beta \in[0,1-\alpha)$.

Proof. Let $\beta \in[0,1-\alpha)$ and $\varepsilon \in(0,1-\alpha-\beta)$ be fixed and let any $\delta \in(0, T-t)$ be given. Denote $\gamma=\alpha+\beta+\varepsilon$.

We now prove the continuity of $\left(A^{*}\right)^{\beta} \psi_{t, x}(\cdot)$ on $[t, T-\delta]$. To show this, we take $t \leq s<\bar{s} \leq T-\delta$ and consider the following:

$$
\begin{aligned}
& \left\|\left(A^{*}\right)^{\beta} \psi_{t, x}(s)-\left(A^{*}\right)^{\beta} \psi_{t, x}(\bar{s})\right\| \\
& \leq\left\|\left(A^{*}\right)^{\alpha+\beta} e^{-A^{*}(T-\bar{s})}\left(I-e^{-A^{*}(\bar{s}-s)}\right) Q_{1} y_{t, x}(T)\right\| \\
& \quad+\int_{s}^{\bar{s}}\left\|\left(A^{*}\right)^{\alpha+\beta} e^{-A^{*}(\sigma-s)} Q y_{t, x}(\sigma)\right\| d \sigma \\
& \quad+\int_{\bar{s}}^{T}\left\|\left(A^{*}\right)^{\alpha+\beta} e^{-A^{*}(\sigma-\bar{s})}\left(I-e^{-A^{*}(\bar{s}-s)}\right) Q y_{t, x}(\sigma)\right\| d \sigma \\
& \triangleq
\end{aligned}
$$

We estimate $I_{1}, I_{2}$ and $I_{3}$ separately. By (1.1), we have

$$
\begin{aligned}
I_{1} & =\left\|\left(A^{*}\right)^{\gamma} e^{-A^{*}(T-\bar{s})}\left(I-e^{-A^{*}(\bar{s}-s)}\right)\left(A^{*}\right)^{-\varepsilon} Q_{1} y_{t, x}(T)\right\| \\
& \leq \frac{c_{\varepsilon} c_{\gamma}}{|T-\bar{s}|^{\gamma}}|\bar{s}-s|^{\varepsilon}\left\|Q_{1} y_{t, x}(T)\right\| .
\end{aligned}
$$


By (1.1), it follows from the first inequality in (4.10) that

$$
\begin{aligned}
I_{2} & \leq c_{\alpha+\beta}\|Q\| \int_{s}^{\bar{s}} \frac{\left\|y_{t, x}(\sigma)\right\|}{|\sigma-s|^{\alpha+\beta}} d \sigma \\
& \leq \int_{s}^{\bar{s}} \frac{K c_{\alpha+\beta}\|Q\|}{|\sigma-s|^{\alpha+\beta}} d \sigma\left[\|x\|+\frac{\left\|Q_{1} y_{t, x}(T)\right\|}{\delta^{\alpha}}+\int_{t}^{T}\left\|y_{t, x}(\tau)\right\| d \tau\right] \\
& \leq \frac{K c_{\alpha+\beta}\|Q\|}{1-\alpha-\beta}|\bar{s}-s|^{1-\alpha-\beta}\left[\|x\|+\frac{\left\|Q_{1} y_{t, x}(T)\right\|}{\delta^{\alpha}}+\int_{t}^{T}\left\|y_{t, x}(\tau)\right\| d \tau\right] .
\end{aligned}
$$

Finally, by (1.1), we have from the second inequality in (4.10), that

$$
\begin{aligned}
I_{3} & =\int_{\bar{s}}^{T}\left\|\left(A^{*}\right)^{\gamma} e^{-A^{*}(\sigma-s)}\left(I-e^{-A^{*}(\bar{s}-s)}\right)\left[\left(A^{*}\right)^{-\varepsilon} Q y_{t, x}(\sigma)\right]\right\| d \sigma \\
& \leq c_{\varepsilon} c_{\gamma}\|Q\||\bar{s}-s|^{\varepsilon} \int_{\bar{s}}^{T} \frac{\left\|y_{t, x}(\sigma)\right\|}{|\sigma-\bar{s}|^{\gamma}} d \sigma \\
& \leq K_{\gamma} c_{\varepsilon} c_{\gamma}\|Q\||\bar{s}-s|^{\varepsilon}\left[\|x\|+\frac{\left\|Q_{1} y_{t, x}(T)\right\|}{\delta^{\alpha}}+\int_{t}^{T}\left\|y_{t, x}(\tau)\right\| d \tau\right] .
\end{aligned}
$$

Combining (4.19)-(4.22) yields $\left(A^{*}\right)^{\beta} \psi_{t, x}(\cdot) \in C([t, T-\delta] ; X)$.

Now we prove the continuity of $y_{t, x}(\cdot)$ on $[t, T-\delta]$ and $A^{\beta} y_{t, x}(\cdot)$ on $(t, T-$ $\delta$ ]. Taking $t<s<\bar{s} \leq T-\delta$, we have from (1.1) and (4.11) for some $C>0$

$$
\begin{aligned}
& \left\|A^{\beta} y_{t, x}(s)-A^{\beta} y_{t, x}(\bar{s})\right\| \leq\left\|A^{\beta} e^{-A(s-t)}\left(I-e^{-A(\bar{s}-s)}\right) x\right\| \\
& \quad+\int_{s}^{\bar{s}}\left\|A^{\alpha+\beta} e^{-A(\bar{s}-r)} B R^{-1} B^{*} \psi_{t, x}(r)\right\| d r \\
& \quad+\int_{t}^{s}\left\|A^{\gamma} e^{-A(s-r)}\left(I-e^{-A(\bar{s}-s)}\right)\left[A^{-\varepsilon} B R^{-1} B^{*} \psi_{t, x}(r)\right]\right\| d r \\
& \leq\left\|\left(I-e^{-A(\bar{s}-s)}\right) A^{\beta} e^{-A(s-t)} x\right\|+C|\bar{s}-s|^{\varepsilon} \int_{t}^{s} \frac{\left\|\psi_{t, x}(r)\right\|}{|s-r|^{\gamma}} d r \\
& \quad+C K \int_{s}^{\bar{s}} \frac{1}{|\bar{s}-r|^{\alpha+\beta}} d r\left[\|x\|+\frac{\left\|Q_{1} y_{t, x}(T)\right\|}{\delta^{\alpha}}+\int_{t}^{T}\left\|\psi_{t, x}(\tau)\right\| d \tau\right] \\
& \leq C\left\|\left(I-e^{-A(\bar{s}-s)}\right) A^{\beta} e^{-A(s-t)} x\right\|+C\left(K_{\gamma}|\bar{s}-s|^{\varepsilon}+K \frac{|\bar{s}-s|^{1-\alpha-\beta}}{1-\alpha-\beta}\right) \\
& \quad\left[\|x\|+\frac{\left\|Q_{1} y_{t, x}(T)\right\|}{\delta^{\alpha}}+\int_{t}^{T}\left\|\psi_{t, x}(\tau)\right\| d \tau\right] .
\end{aligned}
$$


This yields $A^{\beta} y_{t, x}(\cdot) \in C((t, T-\delta] ; X)$. Similarly, we can obtain that $y_{t, x}(\cdot) \in C([t, T-\delta] ; X)$. Since $\delta$ is arbitrary, our conclusion follows.

5. The Fredholm integral equation and the state feedback control. In this section, we prove the equivalence between the two-point boundary value problem and a Fredholm integral equation in Theorem 5.2, which is a modification of a result found in [17, p.390], and then give the state feedback representations of optimal controls without additional hypothesis, which generalizes the results of [17], [22], [24], etc. Afterwards, some properties of solutions and an existence and uniqueness condition for solution to the Fredholm integral equation are also given in Theorems 5.5 and 5.6. These properties will play an important role in Section 6 to derive the closed-loop synthesis via the solution to the Riccati integral equation.

For $t \in[0, T)$ given, define

$$
\left\{\begin{aligned}
S_{0}(s, t) x= & \left(A^{*}\right)^{\alpha} e^{-A^{*}(T-s)} Q_{1} e^{-A(T-t)} x \\
& +\int_{s}^{T}\left(A^{*}\right)^{\alpha} e^{-A^{*}(\sigma-s)} Q e^{-A(\sigma-t)} x d \sigma \\
S_{1}(s, r) x= & \int_{s \vee r}^{T}\left(A^{*}\right)^{\alpha} e^{-A^{*}(\sigma-s)} Q A^{\alpha} e^{-A(\sigma-r)} x d \sigma \\
S_{2}(s, r) x= & \left(A^{*}\right)^{\alpha} e^{-A^{*}(T-s)} Q_{1} A^{\alpha} e^{-A(T-r)} x
\end{aligned}\right.
$$

where $s \vee r=\max (s, r)$. Consider the following equation

$$
H(s, t)=-S_{0}(s, t)-\int_{t}^{T}\left[S_{1}(s, r)+S_{2}(s, r)\right] B R^{-1} B^{*} H(r, t) d r
$$

for $s \in[t, T]$. We refer to (5.2) as the Fredholm integral equation for Problem $(L Q)_{t}$.

Definition 5.1. For $t \in[0, T)$ given, a linear operator $H(\cdot, t): X \mapsto$ $L^{1}(t, T ; X)$, is called a solution to (5.2) if the following holds

$$
\begin{gathered}
H(s, t) x=-S_{0}(s, t) x-\int_{t}^{T}\left[S_{1}(s, r)+S_{2}(s, r)\right] B R^{-1} B^{*} H(r, t) x d r, \\
\text { a.e. } s \in[t, T], \quad \forall x \in X .
\end{gathered}
$$


Theorem 5.2. For $t \in[0, T)$ given.

(i) Suppose a solution $H(\cdot, t)$ to $(5.2)$ satisfies

$$
B^{*} H(\cdot, t) x \in \mathcal{U}[t, T], \quad \int_{t}^{T} A^{\alpha} e^{-A(T-r)} B R^{-1} B^{*} H(r, t) x d r \in X,
$$

then, for any $x \in X$, (4.6) admits a solution $\left(y_{t, x}(\cdot), \psi_{t, x}(\cdot)\right) \in \mathcal{X}[t, T] \times$ $L^{1}(t, T ; X)$ with $(4.5)$ and

$$
\psi_{t, x}(s)=H(s, t) x, \quad \text { a.e } s \in[t, T]
$$

(ii) For any $x \in X$, (4.6) admits a unique solution $\left(y_{t, x}(\cdot), \psi_{t, x}(\cdot)\right) \in$ $\mathcal{X}[t, T] \times L^{1}(t, T ; X)$ with the property (4.5) if and only if (5.2) admits a unique solution $H(\cdot, t)$ satisfying $(5.4)$.

Proof. (i) For any $x \in X$, we define $\psi_{t, x}(\cdot)$ by (5.5) and $y_{t, x}(\cdot)$ through the first equation in (4.6). Due to $(5.4),(4,5)$ holds and $y_{t, x}(T)$ is well defined. From the proof of Lemma 3.6 ( or (3.50) ) in [17, p.388], we have, for the same absolute constant $c_{\alpha}>0$ in (1.1), that

$$
\begin{aligned}
& \int_{t}^{T}\left\|\int_{t}^{T} S_{1}(s, r) B R^{-1} B^{*} \psi_{t, x}(r) d r\right\|^{2} d s \\
& \leq \int_{t}^{T}\left\{\int_{t}^{T} \int_{s \vee r}^{T}\left\|\left(A^{*}\right)^{\alpha} e^{-A^{*}(\sigma-s)} Q A^{\alpha} e^{-A(\sigma-r)} B R^{-1} B^{*} \psi_{t, x}(r)\right\| d \sigma d r\right\}^{2} d s \\
& \leq c_{\alpha}^{2}\|Q\|\left\|B R^{-1}\right\| \int_{t}^{T}\left\{\int_{t}^{T} \int_{s \vee r}^{T}(\sigma-s)^{-\alpha}(\sigma-r)^{-\alpha}\left\|B^{*} \psi_{t, x}(r)\right\| d \sigma d r\right\}^{2} d s \\
& \leq c_{\alpha}^{2}\|Q\|\left\|B R^{-1}\right\| T^{4(1-\alpha)}(1-\alpha)^{-4}\left\|B^{*} \psi_{t, x}\right\|_{\mathcal{U}[t, T]}^{2} .
\end{aligned}
$$

Since

$$
\left(A^{*}\right)^{\alpha} e^{-A^{*}(T-s)} Q_{1} L_{T}(t) R^{-1} B^{*} \psi_{t, x}(\cdot)=\int_{t}^{T} S_{2}(s, r) B R^{-1} B^{*} \psi_{t, x}(r) d r,
$$

it also holds that

$$
\left\|\int_{t}^{T} S_{2}(s, r) B R^{-1} B^{*} \psi_{t, x}(r) d r\right\| \leq \frac{c_{\alpha}\left\|Q_{1}\right\|}{|T-s|^{\alpha}}\left\|L_{T}(t) R^{-1} B^{*} \psi_{t, x}\right\| .
$$


Hence, by (1.1), (5.6), (5.7) and Fubini Theorem, it follows from (5.3)

$$
\begin{aligned}
\psi_{t, x}(s) & =H(s, t) x \\
& =-S_{0}(s, t) x-\int_{t}^{T}\left\{S_{1}(s, r)+S_{2}(s, r)\right\} B R^{-1} B^{*} \psi_{t, x}(r) d r \\
& =-\left(A^{*}\right)^{\alpha} e^{-A^{*}(T-s)} Q_{1} y_{t, x}(T)-\int_{s}^{T}\left(A^{*}\right)^{\alpha} e^{-A^{*}(\sigma-s)} y_{t, x}(\sigma) d \sigma .
\end{aligned}
$$

(ii) Necessity. For any $x \in X$, by the uniqueness of the solution $\left(y_{t, x}(\cdot)\right.$, $\left.\psi_{t, x}(\cdot)\right)$ to $(4.6)$ with $(4.5)$, we can define a linear operator-valued function $H(\cdot, t): X \mapsto L^{1}(t, T ; X)$ by (5.5). By (5.6), (5.7) and Fubini Theorem, we can reverse the procedure in (5.8). Thus, $H(\cdot, s)$ is a solution to $(5.2)$ with (5.4). The uniqueness of solution to (5.2) with (5.4) follows easily from the proved conclusion (i).

Sufficiency. Let $H(\cdot, t)$ be the unique solution to (5.2) with (5.4). It follows from (i) that, for any $x \in X,(4.6)$ admits a solution $\left(y_{t, x}(\cdot), \psi_{t, x}(\cdot)\right)$ with (4.5). Suppose that for some $\bar{x} \in X$, (4.6) admits another solution $(\tilde{y}(s), \tilde{\psi}(s))$ with $(4.5)$. Then $\hat{\psi}(\cdot) \triangleq \psi_{t, x}(\cdot)-\tilde{\psi}(\cdot) \neq 0$ also satisfies (4.5) and

$$
\hat{\psi}(s)=-\int_{t}^{T}\left\{S_{1}(s, r)+S_{2}(s, r)\right\} B R^{-1} B^{*} \hat{\psi}(r) d r .
$$

Fix $x_{0} \in X$ with $x_{0} \neq 0$, then define $\hat{H}(\cdot)$ by $\hat{H}(\cdot)=\lambda \hat{\psi}(s)$, a.e. $s \in[t, T]$, where any $x \in X$ has the unique decomposition $x=\lambda x_{0}+x_{1}$, with $\left(x_{0}, x_{1}\right)=$ 0 and $\lambda \in \mathbb{R}$. So $H(s, t)+\hat{H}(s)$ is also another solution to (5.2) with (5.4), which yields a contradiction.

If Problem $(L Q)_{t}$ admits a unique optimal control $\bar{u}_{t, x}(\cdot)$ at any $x \in X$, then the two-point boundary value problem (4.6) admits a unique solution $\left(\bar{y}_{t, x}(\cdot), \psi_{t, x}(\cdot)\right)$, in which $\bar{y}_{t, x}(\cdot)$ is the optimal state trajectory, and the Fredholm integral equation (5.2) admits a unique solution $H(\cdot, t)$ satisfying (5.4). The uniqueness of solution to (4.6) with (4.5) and Theorem 4.5 imply

$$
\begin{gathered}
\left\{\begin{array}{l}
\bar{y}_{t, x}(s)=\bar{y}_{r, \bar{y}_{t, x}(r)}(s), \\
\psi_{t, x}(s)=\psi_{r, \bar{y}_{t, x}(r)}(s),
\end{array} \quad \forall t \leq r \leq s<T .\right. \\
\psi_{t, x}(r)=\lim _{s \rightarrow r} \psi_{r, \bar{y}_{t, x}(r)}(s)=\lim _{s \rightarrow r} H(s, r) \bar{y}_{t, x}(r)=H(r, r) \bar{y}_{t, x}(r),
\end{gathered}
$$


and

$$
\bar{u}_{t, x}(r)=R^{-1} B^{*} \psi_{t, x}(r)=R^{-1} B^{*} H(r, r) \bar{y}_{t, x}(r), \quad \forall r \in[t, T) .
$$

Thus, we obtain the state feedback representations of the optimal control by the solution to the Fredholm integral equation (5.2).

Let us define that

$$
\begin{aligned}
{[u(\cdot), v(\cdot)] \triangleq } & \left\langle\Phi_{0}(t) u(\cdot), v(\cdot)\right\rangle+\left\langle Q_{1} L_{T}(t) u(\cdot), L_{T}(t) v(\cdot)\right\rangle, \\
& \forall u(\cdot), v(\cdot) \in D\left(L_{T}(t)\right),
\end{aligned}
$$

which is a bilinear functional on $D\left(L_{T}(t)\right)$, and

$$
\begin{aligned}
f_{t}(x, u(\cdot)) \triangleq & \left\langle\Theta_{0}(t) x, u(\cdot)\right\rangle+\left\langle Q_{1} L_{T}(t) u(\cdot), e^{-A(T-t)} x\right\rangle, \\
& \forall u(\cdot) \in D\left(L_{T}(t)\right),
\end{aligned}
$$

which is a linear functional on $D\left(L_{T}(t)\right)$.

Theorem 5.3. (i) Suppose that, for $t \in[0, T)$, the Fredholm integral equation (5.2) admits a unique solution $H(\cdot, t)$ satisfying (5.4). Then

$$
[u(\cdot), u(\cdot)]>0 \quad \forall u(\cdot) \in D\left(L_{T}(t)\right), \quad u(\cdot) \neq 0,
$$

and $\left(D\left(L_{T}(t)\right),[\cdot, \cdot]\right)$ is an inner product space.

Conversely, suppose that (5.15) holds, then the Fredholm integral equation (5.2) admits at most one solution satisfying (5.4).

(ii) Problem $(L Q)_{t}$ is solvable at $x \in X$ with an optimal control $\bar{u}_{t, x}(\cdot)$ if and only if (4.2) holds and

$$
f_{t}(x, u(\cdot))=\left[u(\cdot),-\bar{u}_{t, x}(\cdot)\right], \quad \forall u(\cdot) \in D\left(L_{T}(t)\right) .
$$

Proof. (i) Theorems 4.1 and 5.2 imply (4.2) holds. We first prove that $u(\cdot)=0$, if $[u(\cdot), v(\cdot)]=0$ for all $v(\cdot) \in D\left(L_{T}(t)\right)$. The assumption $[u(\cdot), v(\cdot)]$ $=0$ for all $v(\cdot) \in D\left(L_{T}(t)\right)$ yields that

$$
L_{T}^{*}(t) Q_{1} L_{T}(t) u(\cdot)+\Phi_{0}(t) u(\cdot)=0 .
$$

Therefore,

$L_{T}^{*}(t) Q_{1}\left[L_{T}(t)\left(\bar{u}_{t, x}(\cdot)+u(\cdot)\right)+e^{-A(T-t)} x\right]+\Phi_{0}(t)\left(\bar{u}_{t, x}(\cdot)+u(\cdot)\right)+\Theta_{0} x=0$. 
By Theorem 4.1, $\bar{u}_{t, x}(\cdot)+u(\cdot)$ is also an optimal control for Problem $(L Q)_{t}$ at $x \in X$. The uniqueness of optimal control implies $u(\cdot)=0$. Now we prove that $u(\cdot)=0$ if $[u(\cdot), u(\cdot)]=0$. For any $k \in(-\infty,+\infty)$,

$0 \leq[u(\cdot)+k v(\cdot), u(\cdot)+k v(\cdot)]=k^{2}[v(\cdot), v(\cdot)]+2 k[u(\cdot), v(\cdot)], \quad \forall v(\cdot) \in D\left(L_{T}(t)\right)$

implies that $[u(\cdot), v(\cdot)]=0$ for all $v(\cdot) \in D\left(L_{T}(t)\right)$. Therefore the proved conclusion implies $u(\cdot)=0$. The first part is proved. then

If (5.15) holds, we suppose that (4.1) holds for $u_{1}(\cdot), u_{2}(\cdot) \in D\left(L_{T}(t)\right)$,

$$
L_{T}^{*}(t) Q_{1} L_{T}(t)\left(u_{1}(\cdot)-u_{2}(\cdot)\right)+\Phi_{0}(t)\left(u_{1}(\cdot)-u_{2}(\cdot)\right)=0 .
$$

Thus (5.15) implies $u_{1}(\cdot)=u_{2}(\cdot)$. From Theorem 5.2 (i), Theorems 4.1 and 4.2 , we get that the second part of (i).

(ii) For any $u(\cdot) \in D\left(L_{T}(t)\right)$,

$$
\begin{aligned}
& \left\langle\Theta_{0}(t) x, u(\cdot)\right\rangle+\left\langle Q_{1} L_{T}(t) u(\cdot), e^{-A(T-t)} x\right\rangle \\
& =-\left[\left\langle\Phi_{0}(t) \bar{u}_{t, x}(\cdot), u(\cdot)\right\rangle+\left\langle Q_{1} L_{T}(t) \bar{u}_{t, x}(\cdot), L_{T}(t) u(\cdot)\right\rangle\right],
\end{aligned}
$$

which is equivalent to (4.1), or (5.16). Then Theorem 4.1 applies.

Theorem 5.4. Suppose that $Q_{1}=Q_{0}+\left(A^{*}\right)^{-\beta} E A^{-\beta}$ with $Q_{0} \geq 0$ or $Q_{0} \leq 0, E^{*}=E \in \mathcal{L}(X)$ and $\beta>\alpha-\frac{1}{2}$ is nonnegative, and

$$
[u(\cdot), u(\cdot)] \geq \delta\|u(\cdot)\|_{\mathcal{U}[t, T]}^{2}, \quad \forall u(\cdot) \in D\left(L_{T}(t)\right)
$$

where $\delta>0$ is an absolute constant. If the Fredholm integral equation (5.2) admits a unique solution satisfying (5.4), then Problem $(L Q)_{t}$ is uniquely solvable at any $x \in X$ with the optimal pair $\left(\bar{u}_{t, x}(\cdot), \bar{y}_{t, x}(\cdot)\right)$, which satisfies

$$
\left\|\bar{u}_{t, x}(\cdot)\right\|_{\mathcal{U}[t, T]}, \quad\left\|\bar{y}_{t, x}(\cdot)\right\|_{\mathcal{X}[t, T]}, \quad\left\|Q_{1} \bar{y}_{t, x}(T)\right\|_{X} \leq L\|x\|,
$$

where $L>0$ is absolute constant independent of $x \in X$ and $t \in[0, T)$.

Proof. Let $Q_{0} \geq 0$. From Theorem 5.2 (ii) and Theorem 4.2, it follows that Problem $(L Q)_{t}$ is uniquely solvable at $x \in X$ with the optimal pair $\left(\bar{u}_{t, x}(\cdot), \bar{y}_{t, x}(\cdot)\right)$. Obviously, (5.18) implies that

$$
\begin{aligned}
& \left|\left\langle\Theta_{0}(t) x, u(\cdot)\right\rangle\right| \leq\left\|\Theta_{0}(t) x\right\|_{\mathcal{U}[t, T]}\|u(\cdot)\|_{\mathcal{U}[t, T]} \\
& \leq \frac{\sqrt{T}}{\delta} \max _{t \in[0, T)}\left\|\Theta_{0}(t)\right\|[u(\cdot), u(\cdot)]^{\frac{1}{2}}\|x\| \leq K_{1}[u(\cdot), u(\cdot)]^{\frac{1}{2}}\|x\| .
\end{aligned}
$$


Since $\beta>\alpha-\frac{1}{2}$, by (1.1) and the Hölder inequality, we have

$$
\left\|A^{-\beta} L_{T}(t) u(\cdot)\right\|=\left\|\int_{t}^{T} A^{-\beta+\alpha} e^{-A(T-s)} B u(s) d s\right\| \leq K_{2}\|u(\cdot)\|_{\mathcal{U}[t, T]} .
$$

Since $Q_{0} \geq 0$, and

$$
\left\|Q_{0} x\right\| \leq\left\|Q_{0}^{\frac{1}{2}}\right\|\left\|Q_{0}^{\frac{1}{2}} x\right\| \leq\left\|Q_{0}^{\frac{1}{2}}\right\|\left\{\left\langle Q_{0} x, x\right\rangle\right\}^{\frac{1}{2}}, \quad \forall x \in X,
$$

from (5.18) and (5.21), we obtain that

$$
\begin{aligned}
\left|\left\langle Q_{1} L_{T}(t) u(\cdot), e^{-A(T-t)} x\right\rangle\right| \leq\left\|e^{-A(T-t)}\right\|\left\|Q_{1} L_{T}(t) u(\cdot)\right\|\|x\| \\
\leq K_{3}\left\{\|u(\cdot)\|_{\mathcal{U}[t, T]}+\left\|Q_{0}^{\frac{1}{2}} L_{T}(t) u(\cdot)\right\|\right\}\|x\| \\
=K_{3}\left\{\|u(\cdot)\|_{\mathcal{U}[t, T]}+\left\{\left\langle Q_{0} L_{T}(t) u(\cdot), L_{T}(t) u(\cdot)\right\rangle\right\}^{\frac{1}{2}}\right\}\|x\| \\
=K_{3}\left\{\left\{[u(\cdot), u(\cdot)]-\left\langle\Phi_{0}(t) u(\cdot), u(\cdot)\right\rangle-\left\langle E A^{-\beta} L_{T}(t) u(\cdot), A^{-\beta} L_{T}(t) u(\cdot)\right\rangle\right\}^{\frac{1}{2}}\right. \\
\left.\quad+\|u(\cdot)\|_{\mathcal{U}[t, T]}\right\}\|x\| \\
\leq K_{3}\left\{\left\{[u(\cdot), u(\cdot)]+\left(\max _{t \in[0, T)}\left\|\Phi_{0}(t)\right\|+\|E\| K_{2}^{2}\right)\|u(\cdot)\|_{\mathcal{U}[t, T]}^{2}\right\}^{\frac{1}{2}}\right. \\
\left.\quad+\|u(\cdot)\|_{\mathcal{U}[t, T]}\right\}\|x\| \leq K_{4}[u(\cdot), u(\cdot)]^{\frac{1}{2}}\|x\| .
\end{aligned}
$$

Hence the following holds for $K_{5}=K_{1}+K_{4}$

$$
\begin{aligned}
\left|f_{t}(x, u(\cdot))\right| & =\left|\left\langle\Theta_{0}(t) x, u(\cdot)\right\rangle+\left\langle Q_{1} L_{T}(t) u(\cdot), e^{-A(T-t)} x\right\rangle\right| \\
& \leq K_{5}[u(\cdot), u(\cdot)]^{\frac{1}{2}}\|x\| .
\end{aligned}
$$

Thus, it follows from (5.16) and (5.24) that

$$
\left\|\bar{u}_{t, x}(\cdot)\right\|_{\mathcal{U}[t, T]} \leq \delta^{-\frac{1}{2}}\left[\bar{u}_{t, x}(\cdot), \bar{u}_{t, x}(\cdot)\right]^{\frac{1}{2}}=\delta^{-\frac{1}{2}}\left\|f_{t}(x, \cdot)\right\| \leq \delta^{-\frac{1}{2}} K_{5}\|x\|,
$$

where $\left\|f_{t}(x, \cdot)\right\|$ is the norm of the bounded functional $f_{t}(x, \cdot)$ on $\left(D\left(L_{T}(t)\right)\right.$, $[\cdot, \cdot])$. Similar to the proof of $(5.23)$, we obtain from $(5.25)$ that

$$
\begin{aligned}
& \left\|Q_{1} L_{T}(t) \bar{u}_{t, x}(\cdot)\right\| \leq K_{6}\left\{\left\|Q_{0}^{\frac{1}{2}} L_{T}(t) \bar{u}_{t, x}(\cdot)\right\|+\left\|\bar{u}_{t, x}(\cdot)\right\|_{\mathcal{U}[t, T]}\right\} \\
& \leq K_{7}\left\{\left[\bar{u}_{t, x}(\cdot), \bar{u}_{t, x}(\cdot)\right]+\left\|\bar{u}_{t, x}(\cdot)\right\|_{\mathcal{U}[t, T]}^{2}\right\}^{\frac{1}{2}} \leq K_{7}\left(\frac{1+\delta}{\delta}\right)^{\frac{1}{2}}\left[\bar{u}_{t, x}(\cdot), \bar{u}_{t, x}(\cdot)\right]^{\frac{1}{2}} \\
& \leq K_{5} K_{7}(1+\delta)^{\frac{1}{2}} \delta^{-1}\|x\| .
\end{aligned}
$$


This yields

$$
\left\|Q_{1} \bar{y}_{t, x}(T)\right\|=\left\|Q_{1} e^{-A(T-t)} x+Q_{1} L_{T}(t) \bar{u}_{t, x}(\cdot)\right\| \leq K_{8}\|x\| .
$$

Finally we obtain from Lemma 1.1 in $[17$, p.363] and (5.25) that

$$
\left\|\bar{y}_{t, x}(\cdot)\right\|_{\mathcal{U}[t, T]} \leq K_{9}\left(\|x\|+\left\|\bar{u}_{t, x}(\cdot)\right\|_{\mathcal{U}[t, T]}\right) \leq K_{10}\|x\| .
$$

From the above procedure, we can consider all constants $K_{i}(i=1, \ldots, 10)$ to be independent of $t \in[0, T)$. Thus (5.19) follows. The case $Q_{0} \leq 0$ can be similarly considered.

Theorem 5.5. Suppose that the assumption of Theorem 5.4 holds, and $Q_{0}^{\frac{1}{2}} L_{1}(t)$ or $\left(-Q_{0}\right)^{\frac{1}{2}} L_{1}(t)$ is closed respectively. Then the Fredholm integral equation (5.2) admits a unique solution $H(\cdot, t)$ satisfying $(5.4)$, and for any $x \in X$, Problem $(L Q)_{t}$ is uniquely solvable with the optimal pair $\left(\bar{u}_{t, x}(\cdot), \bar{y}_{t, x}(\cdot)\right)$ satisfying $(5.19)$.

Proof. It follows from $(5.18)$ that $\left(D\left(L_{T}(t)\right),[\cdot, \cdot]\right)$ is an inner product space. $(5.18)$ and the closedness of $Q_{0}^{\frac{1}{2}} L_{T}(t)$ or $\left(-Q_{0}\right)^{\frac{1}{2}} L_{T}(t)$ imply that $\left(D\left(L_{T}(t)\right)\right.$, $[\cdot, \cdot])$ is a Hilbert space. By inequality $(5.24)$, we have that $f_{t}(x, u(\cdot))$ is a bounded functional on $\left(D\left(L_{T}(t)\right),[\cdot, \cdot]\right)$ for any $x \in X$. Riesz Represent Theorem and Theorem 5.3 (ii) imply that Problem $(L Q)_{t}$ is solvable at any $x \in X$. (5.18) and Theorem 5.3 (i) yields the uniqueness. The rest follows from Theorems 4.2, 5.2 and 5.4 .

Remark 5.6. If $E=0$, then the assumption of Theorem 5.5 is the same as those in $[15,16]$ except for $Q$ and $Q_{1}$ allowed to be indefinite and non-positive respectively here. For the special case $0 \leq \alpha<\frac{1}{2}$, if $Q_{0}=0$ and $\beta=0$, then both $Q_{1}=E$ and $Q$ are allowed to be indefinite. Therefore, the assumption of Theorem 5.5 is more general than one in $[15,16]$. Theorem 5.5 tells us that under proper conditions, the Fredholm integral equation (5.2) admits a unique solution $H(\cdot, t)$ for $t \in[0, T)$ satisfying (5.4) and our optimal control $\bar{u}_{t, x}(\cdot)$ can be written as a state feedback form (5.12). The assumption that $Q_{0}^{\frac{1}{2}} L_{1}(t)$ or $\left(-Q_{0}\right)^{\frac{1}{2}} L_{1}(t)$ is closed can not be dispensed with, for otherwise the optimal control may not exist (see $[9,15,16])$.

6. Riccati integral equation. In this section, we derive the Riccati integral equation from the Fredholm integral equation from the results in 
the previous section and the closed-loop synthesis of the optimal control determined by the Riccati operator. We will prove that the Riccati operator is also a solution to the Riccati differential equation in the weak sense, and also establish several regularity properties and an asymptotic property of $P(\cdot)$ in the strong sense as $t \rightarrow T$. This is stronger than the existing results, for example, (2.23) in [15], (1.29) in [16] and (4.14) in [17, p.397], which were only in the weak sense. A simple explanatory example is given as an application of our results at the end.

We define by $C_{s}([0, T) ; \mathcal{L}(X))$ the set of all strong continuous mapping $F:[0, T) \mapsto \mathcal{L}(X)$, that is, $F(\cdot) x$ is continuous for any $x \in X$.

Definition 6.1. The integral equation of $P(\cdot) \in C_{s}([0, T) ; \mathcal{L}(X))$

$$
\begin{aligned}
P(t) & =e^{-A^{*}(T-t)} Q_{1} e^{-A(T-t)}+\int_{t}^{T} e^{-A^{*}(\sigma-t)} Q e^{-A(\sigma-t)} d \sigma \\
& -\int_{t}^{T} e^{-A^{*}(\sigma-t)}\left[\left(A^{*}\right)^{\alpha} P(\sigma)\right]^{*} B R^{-1} B^{*}\left(A^{*}\right)^{\alpha} P(\sigma) e^{-A(\sigma-t)} d \sigma,
\end{aligned}
$$

is called a Riccati integral equation. If $P(\cdot)$ satisfies (6.1) in the strong sense, then $P(\cdot)$ is called a solution to the Riccati integral equation $(6.1)$ on $[0, T)$.

If the Fredholm integral equation (5.2) admits a unique solution $H(\cdot, t)$ satisfying (5.4) for any $t \in[0, T)$, then Theorem 5.2 implies Problem $(L Q)_{t}$ admits a unique optimal control $\bar{u}_{t, x}(\cdot)$, and Theorem 4.5 implies the continuity of the optimal pair $\left(\bar{u}_{t, x}(\cdot), \bar{y}_{t, x}(\cdot)\right)$ on $[t, T)$ for all $x \in X$ and $t \in[0, T)$. We can define the operator $S(\cdot, \cdot)$ as follows:

$$
S(s, t) x=\bar{y}_{t, x}(s), \quad \forall 0 \leq t \leq s<T, \quad x \in X .
$$

Due to the uniqueness of the optimal trajectory $\bar{y}_{t, x}$, we must have

$$
S(t, t) x=I, \quad S(s, t)=S(s, r) S(r, t), \quad \forall 0 \leq t \leq r \leq s<T .
$$

Theorem 6.2. Suppose that the assumption of Theorem 5.4 hold. If the Fredholm integral equation (5.2) admits a unique solution $H(\cdot, t)$ for any $t \in$ $[0, T)$ satisfying (5.4), then for any $x \in X$, both the mapping $(s, t) \mapsto S(s, t) x$ and $(s, t) \mapsto H(s, t) x$ are continuous on the set $\{(s, t): 0 \leq t \leq s<T\}$; for some absolute constant $C>0$, it hold that

$$
\left\{\begin{array}{l}
\|S(s, t)\| \leq \frac{C}{|T-s|^{\alpha}}, \quad \forall 0 \leq t \leq s<T . \\
\left\|Q_{1} S(T, t)\right\| \leq C,
\end{array}\right.
$$


and for some constant $C(\gamma)>0$, it hold that

$$
\left\|\left(A^{*}\right)^{\gamma-\alpha} H(s, t)\right\| \leq \frac{C(\gamma)}{|T-s|^{\gamma}} \quad \forall 0 \leq t \leq s<T, \quad 0 \leq \gamma<1 .
$$

Moreover, the operator $P(\cdot)$ defined by

$$
P(t)=-\left(A^{*}\right)^{-\alpha} H(t, t), \quad \forall t \in[0, T),
$$

is a solution to the Riccati integral equation (6.1), which satisfies $P^{*}(t)=$ $P(t) \in \mathcal{L}(X)$ for all $t \in[0, T),\left(A^{*}\right)^{\gamma} P(\cdot) \in C_{s}([0, T) ; \mathcal{L}(X))$ for $\gamma \in[0,1)$ and

$$
\begin{gathered}
\left\|\left(A^{*}\right)^{\gamma} P(t)\right\| \leq \frac{C(\gamma)}{|T-t|^{\gamma}}, \quad\|P(t)\| \leq C, \quad \forall t \in[0, T), \gamma \in[0,1) ; \\
\langle P(t) x, x\rangle=J_{t, x}\left(\bar{u}_{t, x}(\cdot)\right), \quad \forall x \in X, \quad t \in[0, T) .
\end{gathered}
$$

Proof. From (5.19) and (4.10), we obtain (6.4), and from (5.19), (5.5) and (4.12) we obtain (6.5). We now prove the continuity of $(s, t) \mapsto S(s, t) x$ and $(s, t) \mapsto H(s, t) x$ on the set $\{(s, t): 0 \leq t \leq s<T\}$. To this end, let us take $0 \leq t<\bar{t} \leq s<T$ and $x \in X$. Since (1.1), (6.3), (6.4) and (6.5), from Theorem 4.4, 4.5 and 5.4, we see that

$$
\begin{aligned}
& \|S(s, \bar{t}) x-S(s, t) x\|=\|S(s, \bar{t})(I-S(\bar{t}, t)) x\| \\
& \leq \frac{C}{|T-s|^{\alpha}}\left\|\left(e^{-A(\bar{t}-t)}-I\right) x+\int_{t}^{\bar{t}} A^{\alpha} e^{-A(\bar{t}-r)} B R^{-1} B^{*} \psi_{t, x}(r) d r\right\| \\
& \leq \frac{C}{|T-s|^{\alpha}}\left\{\left\|\left(e^{-A(\bar{t}-t)}-I\right) x\right\|+\int_{t}^{\bar{t}} \frac{c_{\alpha} C(0)\left\|B R^{-1} B^{*}\right\|\|x\|}{|\bar{t}-r|^{\alpha}|T-r|^{\alpha}} d r \|\right\} \\
& \leq \frac{C_{1}}{|T-s|^{\alpha}}\left\{\left\|\left(e^{-A(\bar{t}-t)}-I\right) x\right\|+\frac{|\bar{t}-t|^{1-\alpha}\|x\|}{|T-s|^{\alpha}}\right\},
\end{aligned}
$$

where $C_{1}=C \max \left\{1, \frac{c_{\alpha} C(0)\left\|B R^{-1} B^{*}\right\|}{1-\alpha}\right\}$. By (5.10), we have

$$
\begin{aligned}
& \|H(s, \bar{t}) x-H(s, t) x\|=\left\|\psi_{\bar{t}, x}(s)-\psi_{t, x}(s)\right\|=\left\|\psi_{\bar{t}, x}(s)-\psi_{\bar{t}, \bar{y}_{t, x}(\bar{t})}(s)\right\| \\
& =\left\|H(s, \bar{t}) x-H(s, \bar{t}) \bar{y}_{t, x}(\bar{t})\right\| \leq\|H(s, \bar{t})\|\|(I-S(\bar{t}, t)) x\| \\
& \leq \frac{C_{2}}{|T-s|^{\alpha}}\left\{\left\|\left(e^{-A(\bar{t}-t)}-I\right) x\right\|+\frac{|\bar{t}-t|^{1-\alpha}\|x\|}{|T-s|^{\alpha}}\right\}
\end{aligned}
$$


where $C_{2}=C(0) \max \left\{1, \frac{c_{\alpha} C(0)\left\|B R^{-1} B^{*}\right\|}{1-\alpha}\right\}$. Combining (6.9), (6.10) with Theorem 4.5, we obtain the continuity of $(s, t) \mapsto S(s, t) x$ and $(s, t) \mapsto$ $H(s, t) x .\left(A^{*}\right)^{\gamma} P(\cdot) \in C_{s}([0, T) ; \mathcal{L}(X))$ for $\gamma \in[0,1)$ follows from $(6.6)$ and Theorem 4.5. The rest follows from $(4.17)$, (4.19)-(4.23) in [17, pp.398400].

Theorem 6.3. If $P(\cdot)$ is a solution to the Riccati integral equation (6.1) on $[0, T)$ which satisfies $\|P(t)\| \leq C$ for some absolute constant $C>0$, then it holds that

$$
\lim _{\tau \rightarrow T}\langle P(\tau) x, y\rangle=\left\langle Q_{1} x, y\right\rangle \quad \forall x, y \in X,
$$

and

$$
\lim _{t \rightarrow T} P(t) x=Q_{1} x \quad \forall x \in X
$$

Proof. Denote

$$
\left\{\begin{array}{l}
N(t)=B^{*}\left(A^{*}\right)^{\alpha} P(t) ; \quad N^{*}(t)=\left[\left(A^{*}\right)^{\alpha} P(t)\right]^{*} B, \\
E(t)=\int_{t}^{T} e^{-A^{*}(\sigma-t)} N^{*}(\sigma) R^{-1} N(\sigma) e^{-A(\sigma-t)} d \sigma,
\end{array} \text { for } t \in[0, T) .\right.
$$

Define $F(t): X \rightarrow \mathcal{U}$ as follows:

$$
[F(t) x](\cdot)=R^{-\frac{1}{2}} N(\cdot) e^{-A(\cdot-t)} x \quad \forall x \in X, \quad t \in[0, T),
$$

and its adjoint operator $F^{*}(t): \mathcal{U} \rightarrow X$ is given by

$$
F^{*}(t) u(\cdot)=\int_{t}^{T} e^{-A^{*}(\sigma-t)} N^{*}(\sigma) R^{-\frac{1}{2}} u(\sigma) d \sigma \quad \forall u(\cdot) \in \mathcal{U}, \quad t \in[0, T) .
$$

Obviously, for $t \in[0, T)$, we have $E(t)=F^{*}(t) F(t)$ and

$$
\|E(t)\|=\left\|F^{*}(t) F(t)\right\|=\|F(t)\|^{2}=\left\|F^{*}(t)\right\|^{2} .
$$

Since $\|P(t)\| \leq C$ for $t \in[0, T)$ and (6.16), the third term in the right hand side of (6.1) implies

$$
\int_{t}^{T}\left\|R^{-\frac{1}{2}} N(\sigma) e^{-A(\sigma-t)} x\right\|^{2} d \sigma<+\infty, \quad \forall x \in X, \quad t \in[0, T),
$$


and it holds that, for some absolute constant $C_{1}>0$,

$$
\|E(t)\| \leq C_{1}, \quad\left\|F^{*}(t)\right\|=\|F(t)\| \leq C_{1}^{\frac{1}{2}} \quad t \in[0, T) .
$$

For any given $x, y \in X$ and $s, t \in[0, T)$, we have from (6.17) that

$$
\begin{aligned}
& \left|\left\langle E(\tau) e^{-A(\tau-s)} x, e^{-A(\tau-t)} y\right\rangle\right|^{2} \\
\leq & \int_{\tau}^{T}\left\|R^{-\frac{1}{2}} N(\sigma) e^{-A(\sigma-s)} x\right\|^{2} d \sigma \int_{\tau}^{T}\left\|R^{-\frac{1}{2}} N(\sigma) e^{-A(\sigma-t)} y\right\|^{2} d \sigma \\
\rightarrow & 0, \quad \text { as } \quad \tau \rightarrow T .
\end{aligned}
$$

Therefore, (6.19) and (6.1) imply

$$
\lim _{\tau \rightarrow T}\left\langle P(\tau) e^{-A(\tau-s)} x, e^{-A(\tau-t)} y\right\rangle=\left\langle Q_{1} e^{-A(T-s)} x, e^{-A(T-t)} y\right\rangle
$$

On the other hand, we have

$$
\begin{aligned}
& \left|\left\langle P(\tau) e^{-A(\tau-s)} x, e^{-A(\tau-t)} y\right\rangle-\left\langle P(\tau) e^{-A(T-s)} x, e^{-A(T-t)} y\right\rangle\right| \\
& \leq\left|\left\langle P(\tau) e^{-A(\tau-s)} x, e^{-A(T-t)} y-e^{-A(\tau-t)} y\right\rangle\right| \\
& \quad+\left|\left\langle e^{-A(T-s)} x-e^{-A(\tau-s)} x, P(\tau) e^{-A(T-t)} y\right\rangle\right| \rightarrow 0, \text { as } \tau \rightarrow T .
\end{aligned}
$$

Thus, it follows from (6.20) and (6.21) that

$$
\lim _{\tau \rightarrow T}\left\langle P(\tau) e^{-A(T-s)} x, e^{-A(T-t)} y\right\rangle=\left\langle Q_{1} e^{-A(T-s)} x, e^{-A(T-t)} y\right\rangle .
$$

Since $\left\{e^{-A(T-t)} y: y \in X\right.$ and $\left.t \in[0, T)\right\}$ is dense in $X$, we deduce from (6.22) and $\|P(t)\| \leq C$ for $t \in[0, T)$ that

$$
\lim _{\tau \rightarrow T}\left\langle P(\tau) e^{-A(T-s)} x, z\right\rangle=\left\langle Q_{1} e^{-A(T-s)} x, z\right\rangle \quad \forall x, z \in X .
$$

Similarly, from the density of $\left\{e^{-A(T-s)} x: x \in X\right.$ and $\left.s \in[0, T)\right\}$ in $X$, $Q_{1}^{*}=Q_{1}$ and $P^{*}(\tau)=P(\tau)$ for $\tau \in[0, T)$, we get (6.11), which is equivalent to

$$
\lim _{\tau \rightarrow T}\langle E(\tau) x, y\rangle=0, \quad \forall x, y \in X
$$


Therefore, for any $x \in X$

$$
\begin{aligned}
& \|E(t) x\|=\left\|F^{*}(t) F(t) x\right\| \leq\left\|F^{*}(t)\right\|\|F(t) x\| \\
& \leq C_{1}{ }^{\frac{1}{2}}\|F(t) x\|=C_{1}{ }^{\frac{1}{2}}\langle E(t) x, x\rangle^{\frac{1}{2}} \rightarrow 0 \quad \text { as } t \rightarrow T .
\end{aligned}
$$

(6.23) and (6.1) yields (6.12) immediately.

Remark 6.4. [15], [16, p.452] and [17, p.397] derived the property (6.11) through the LQ problem (see (2.23) in [15], (1.29) in [16] and (4.14) in [17, p.397]). We know from Theorem 6.2 that the Riccati operator is uniformly bounded if the LQ problem is uniquely solvable at any $x \in X$. In Theorem 6.3 , we directly prove the same property (6.11) only under the assumption of the uniform boundedness of solution to the Riccati integral equation (6.1) and obtain even stronger result (6.12). In fact, the weak form (6.11) and the strong one (6.12) are equivalent. Obviously, (6.12) implies (6.11). On the other hand, (6.11) and Principle of Uniform Boundedness imply (6.18), then it follows from (6.23) and (6.1) that (6.12) holds. Corollary 5.3 in [16, p.470] obtained the strong form (6.12) under the assumption $Q_{1} \geq 0$ and $Q_{1}^{\frac{1}{2}}$ is compact. Here we remove this assumption.

Theorem 6.5. If $P(\cdot) \in C_{s}([0, T) ; \mathcal{L}(X))$ is a solution to the Riccati integral equation (6.1) with $\left(A^{*}\right)^{\alpha} P(\cdot) \in C_{s}([0, T) ; \mathcal{L}(X))$, then $P(t)$ satisfies the following Riccati differential equation on $[0, T)$,

$$
\begin{aligned}
\langle\dot{P}(t) x, y\rangle & =-\langle Q x, y\rangle+\langle P(t) A x, y\rangle+\langle P(t) A y, x\rangle \\
& +\left\langle R^{-1} B^{*}\left(A^{*}\right)^{\alpha} P(t) x, B^{*}\left(A^{*}\right)^{\alpha} P(t) y\right\rangle, \quad \forall x, y \in D(A) .
\end{aligned}
$$

Moreover, if $\left(A^{*}\right)^{\theta} P(\cdot) \in C_{s}([0, T) ; \mathcal{L}(X))$ for some $\theta \in[\alpha, 1)$, then $P(t)$ satisfies the Riccati differential equation (6.24) on $[0, T)$ in the sense that

$$
\begin{aligned}
\langle\dot{P}(t) x, y\rangle & =-\langle Q x, y\rangle+\left\langle\overline{P(t) A^{\theta}} A^{1-\theta} x, y\right\rangle+\left\langle\overline{P(t) A^{\theta}} A^{1-\theta} y, x\right\rangle \\
& +\left\langle R^{-1} B^{*}\left(A^{*}\right)^{\alpha} P(t) x, B^{*}\left(A^{*}\right)^{\alpha} P(t) y\right\rangle, \quad \forall x, y \in D\left(A^{1-\theta}\right),
\end{aligned}
$$

where $\overline{P(t) A^{\theta}}=\left[\left(A^{*}\right)^{\theta} P(t)\right]^{*}$ is the unique extension of $P(t) A^{\theta}$ in $\mathcal{L}(X)$.

Proof. Let $N(\cdot), E(\cdot)$ be defined in (6.13). We only need to prove the second part of this theorem. It follows from $\left(A^{*}\right)^{\alpha} P(\cdot) \in C_{s}([0, T) ; \mathcal{L}(X))$ 
that for any $x, y \in X$ and $t \in[0, T)$,

$$
\left\{\begin{aligned}
& \frac{-1}{\bar{t}-t} \int_{t}^{\bar{t}}\left\langle R^{-1} N(\sigma) e^{-A(\sigma-t)} x, N(\sigma) e^{-A(\sigma-t)} y\right\rangle d \sigma \\
& \rightarrow-\left\langle R^{-1} N(t) x, N(t) y\right\rangle \quad \text { as } \bar{t} \rightarrow t^{+} \\
& \frac{1}{\bar{t}-t} \int_{\bar{t}}^{t}\left\langle R^{-1} N(\sigma) e^{-A(\sigma-\bar{t})} x, N(\sigma) e^{-A(\sigma-\bar{t})} y\right\rangle d \sigma \\
& \rightarrow-\left\langle R^{-1} N(t) x, N(t) y\right\rangle \quad \text { as } \bar{t} \rightarrow t^{-}
\end{aligned}\right.
$$

We obtain from $\left(A^{*}\right)^{\theta} P(\cdot) \in C_{s}([0, T) ; \mathcal{L}(X))$ and $(6.1)$ that $\left(A^{*}\right)^{\theta} E(\cdot) \in$ $C_{s}([0, T) ; \mathcal{L}(X))$. Then, for any $x, y \in D\left(A^{1-\theta}\right)$ and $t \in[0, T)$,

$$
\begin{aligned}
& \frac{1}{\bar{t}-t}\{\langle E(\bar{t}) x, y\rangle-\langle E(t) x, y\rangle\} \\
& =\frac{1}{\bar{t}-t}\left\{\left\langle E(\bar{t})\left(I-e^{-A(\bar{t}-t)}\right) x, y\right\rangle+\left\langle E(\bar{t}) e^{-A(\bar{t}-t)} x,\left(I-e^{-A(\bar{t}-t)}\right) y\right\rangle\right\} \\
& =\frac{1}{\bar{t}-t}\left\{\left\langle\left(I-e^{-A(\bar{t}-t)}\right) A^{-\theta} x,\left(A^{*}\right)^{\theta} E(\bar{t}) y\right\rangle\right. \\
& \left.+\left\langle\left(A^{*}\right)^{\theta} E(\bar{t}) e^{-A(\bar{t}-t)} x,\left(I-e^{-A(\bar{t}-t)}\right) A^{-\theta} y\right\rangle\right\} \\
& \rightarrow\left\langle A^{1-\theta} x,\left(A^{*}\right)^{\theta} E(t) y\right\rangle+\left\langle\left(A^{*}\right)^{\theta} E(t) x, A^{1-\theta} y\right\rangle \text { as } \bar{t} \rightarrow t^{+} ; \\
& \frac{1}{\bar{t}-t}\{\langle E(\bar{t}) x, y\rangle-\langle E(t) x, y\rangle\} \\
& =\frac{1}{\bar{t}-t}\left\{\left\langle\left(e^{A(\bar{t}-t)}-I\right) A^{-\theta} x,\left(A^{*}\right)^{\theta} E(t) e^{A(\bar{t}-t)} y\right\rangle\right. \\
& \left.+\left\langle\left(A^{*}\right)^{\theta} E(t) x,\left(e^{A(\bar{t}-t)}-I\right) A^{-\theta} y\right\rangle\right\} \\
& \rightarrow\left\langle A^{1-\theta} x,\left(A^{*}\right)^{\theta} E(t) y\right\rangle+\left\langle\left(A^{*}\right)^{\theta} E(t) x, A^{1-\theta} y\right\rangle \text { as } \bar{t} \rightarrow t^{-} . \\
& \frac{d}{d t}\left\langle e^{-A^{*}(T-t)} Q_{1} e^{-A(T-t)} x, y\right\rangle=\left\langle A^{1-\theta} x,\left(A^{*}\right)^{\theta} e^{-A^{*}(T-t)} Q_{1} e^{-A(T-t)} y\right\rangle \\
& +\left\langle\left(A^{*}\right)^{\theta} e^{-A^{*}(T-t)} Q_{1} e^{-A(T-t)} x, A^{1-\theta} y\right\rangle ; \\
& \frac{d}{d t} \int_{t}^{T}\left\langle e^{-A^{*}(\sigma-t)} Q e^{-A(\sigma-t)} x, y\right\rangle d \sigma \\
& =-\langle Q x, y\rangle+\int_{t}^{T}\left\langle A^{1-\theta} x,\left(A^{*}\right)^{\theta} e^{-A^{*}(\sigma-t)} Q e^{-A(\sigma-t)} y\right\rangle d \sigma \\
& +\int_{t}^{T}\left\langle\left(A^{*}\right)^{\theta} e^{-A^{*}(\sigma-t)} Q e^{-A(\sigma-t)} x, A^{1-\theta} y\right\rangle d \sigma .
\end{aligned}
$$


Thus, for any $x, y \in D\left(A^{1-\theta}\right)$, we can differentiate the following

$$
\begin{aligned}
& \left\langle e^{-A^{*}(T-t)} Q_{1} e^{-A(T-t)} x, y\right\rangle+\int_{t}^{T}\left\langle e^{-A^{*}(\sigma-t)} Q e^{-A(\sigma-t)} x, y\right\rangle d \sigma \\
& -\int_{t}^{T}\left\langle e^{-A^{*}(\sigma-t)}\left[\left(A^{*}\right)^{\alpha} P(\sigma)\right]^{*} B R^{-1} B^{*}\left(A^{*}\right)^{\alpha} P(\sigma) e^{-A(\sigma-t)} x, y\right\rangle d \sigma \\
& =\langle P(t) x, y\rangle
\end{aligned}
$$

in $t$ to obtain

$$
\begin{aligned}
\langle\dot{P}(t) x, y\rangle= & -\langle Q x, y\rangle+\left\langle A^{1-\theta} x,\left(A^{*}\right)^{\theta} P(t) y\right\rangle+\left\langle\left(A^{*}\right)^{\theta} P(t) x, A^{1-\theta} y\right\rangle \\
& +\left\langle R^{-1} B^{*}\left(A^{*}\right)^{\alpha} P(t) x, B^{*}\left(A^{*}\right)^{\alpha} P(t) y\right\rangle .
\end{aligned}
$$

(6.24) and (6.25) follows immediately.

Theorem 5.5, 6.2, 6.3 and 6.5 imply the following results:

Theorem 6.6. Suppose that the assumption of Theorem 5.5 holds. Then the Riccati integral equation (6.1) has a solution $P(\cdot)$ on $[0, T)$ defined by (6.6), which satisfies $P^{*}(t)=P(t) \in \mathcal{L}(X)$ for all $t \in[0, T),\left(A^{*}\right)^{\gamma} P(\cdot) \in$ $C_{s}([0, T) ; \mathcal{L}(X))$ for any $\gamma \in[0,1),(6.7),(6.8),(6.12)$ and $(6.25)$ for any $\theta \in[0,1)$. Moreover, the unique optimal pair $\left(\bar{u}_{t, x}(\cdot), \bar{y}_{t, x}(\cdot)\right)$ of Problem $(L Q)_{t}$ satisfies

$$
\begin{gathered}
\left\{\begin{array}{l}
\lim _{t \rightarrow T}\left\langle Q_{1} \bar{y}_{t, x}(T), z\right\rangle=\left\langle Q_{1} x, z\right\rangle, \quad \forall z \in X ; \\
\lim _{t \rightarrow T}\left\langle Q_{0}^{\frac{1}{2}} \bar{y}_{t, x}(T), z\right\rangle=\left\langle Q_{0}^{\frac{1}{2}} x, z\right\rangle, \\
o r \lim _{t \rightarrow T}\left\langle\left(-Q_{0}\right)^{\frac{1}{2}} \bar{y}_{t, x}(T), z\right\rangle=\left\langle\left(-Q_{0}\right)^{\frac{1}{2}} x, z\right\rangle,
\end{array} \quad \forall z \in X ;\right. \\
\left\{\begin{array}{l}
\left\|\bar{u}_{t, x}(s)\right\| \leq \frac{L}{(T-s)^{\alpha}}\|x\|, \quad \forall s \in[0, T) ; \\
\left\|Q_{0}^{\frac{1}{2}} \bar{y}_{t, x}(T)\right\|_{X} \leq L\|x\|, \quad \text { or }\left\|\left(-Q_{0}\right)^{\frac{1}{2}} \bar{y}_{t, x}(T)\right\|_{X} \leq L\|x\| ; \\
\left\|\bar{y}_{t, x}(\cdot)\right\|_{C([t, T] ; X)} \leq L\|x\|, \quad \text { if } 0 \leq \alpha<\frac{1}{2} ; \\
\left\|\bar{y}_{t, x}(s)\right\| \leq \frac{L_{\varepsilon}}{(T-s)^{2 \alpha-1+\varepsilon}}\|x\|, \quad \forall s \in[0, T), \quad \text { if } \frac{1}{2} \leq \alpha<1, \quad \varepsilon>0 .
\end{array}\right.
\end{gathered}
$$


where $L, L_{\varepsilon}>0$ are constants independent of $x \in X$ and $t \in[0, T)$ but $L_{\varepsilon}$ dependent of $\varepsilon$.

Proof. The first inequality of (6.34) follows form (4.11) and (5.19). The second inequality of (6.34) follows from (5.22). The third inequality of (6.34) follows from (1.2) and (5.19). If $\frac{1}{2} \leq \alpha<1$ and $0<\varepsilon \leq 1-\alpha$, we have from (1.1) that

$$
\begin{aligned}
& \left\|\left[L(t) \bar{u}_{t, x}(\cdot)\right](s)\right\| \leq c_{\alpha}\|B\|\left\|(T-\cdot)^{\alpha} \bar{u}_{t, x}(\cdot)\right\| \int_{t}^{s} \frac{1}{|s-\tau|^{\alpha}|T-\tau|^{\alpha}} d \tau \\
& \leq c_{\alpha}\|B\|\left\|(T-\cdot)^{\alpha} \bar{u}_{t, x}(\cdot)\right\| \int_{t}^{s} \frac{1}{|s-\tau|^{\alpha}|T-\tau|^{1-\alpha-\varepsilon}|T-\tau|^{2 \alpha-1+\varepsilon}} d \tau \\
& \leq c_{\alpha}\|B\|\left\|(T-\cdot)^{\alpha} \bar{u}_{t, x}(\cdot)\right\| \frac{1}{|T-s|^{2 \alpha-1+\varepsilon}} \int_{t}^{s} \frac{1}{|s-\tau|^{\alpha}|T-\tau|^{1-\alpha-\varepsilon}} d \tau \\
& =\frac{c_{\alpha}\|B\||s-t|^{\varepsilon}}{\varepsilon|T-s|^{2 \alpha-1+\varepsilon}}\left\|(T-\cdot)^{\alpha} \bar{u}_{t, x}(\cdot)\right\|,
\end{aligned}
$$

which yields the last inequality of (6.34) for $0<\varepsilon \leq 1-\alpha$. If $\varepsilon>1-\alpha$, then

$$
\left\|(T-\cdot)^{2 \alpha-1+\varepsilon} \bar{y}_{t, x}(\cdot)\right\| \leq T^{\varepsilon-1+\alpha}\left\|(T-\cdot)^{2 \alpha-1+(1-\alpha)} \bar{y}_{t, x}(\cdot)\right\| .
$$

Thus, the last inequality of (6.34) follows. Since $Q_{0}^{\frac{1}{2}} L_{T}(t)$ is closed, it follows from [12] that $L_{T}^{*}(t) Q_{0}^{\frac{1}{2}}$ is also densely-defined and closed. On the other hand, (5.19) yields that, for $z \in D\left(L_{T}^{*}(t) Q_{0}^{\frac{1}{2}}\right)$,

$$
\begin{aligned}
& \left|\left\langle Q_{0}^{\frac{1}{2}} L_{T}(t) \bar{u}_{t, x}(\cdot), z\right\rangle\right| \leq\left\|\bar{u}_{t, x}(\cdot)\right\|_{\mathcal{U}[t, T]}\left\|L_{T}^{*}(t) Q_{0}^{\frac{1}{2}} z\right\|_{\mathcal{U}[t, T]} \\
& \leq L\|x\|\left\|L_{T}^{*}(t) Q_{0}^{\frac{1}{2}} z\right\|_{\mathcal{U}[t, T]} \leq L\|x\|\left\{\int_{t}^{T}\left\|B^{*}\left(A^{*}\right)^{\alpha} e^{-A^{*}(T-\tau)} Q_{0}^{\frac{1}{2}} z\right\|^{2} d \tau\right\}^{\frac{1}{2}} \\
& \rightarrow 0 \quad \text { as } t \rightarrow T .
\end{aligned}
$$

Therefore, the denseness of $D\left(L_{T}^{*}(t) Q_{0}^{\frac{1}{2}}\right)$ in $X$ and (5.19) imply the second equality of (6.34), which yields the first equality of (6.34). The case of $Q_{0} \leq 0$ can be similarly considered.

Under the assumption of Theorem 6.6, the unique optimal control $\bar{u}(\cdot)$ can also be written as

$$
\bar{u}(s)=-R^{-1} B^{*}\left(A^{*}\right)^{\alpha} P(s) \bar{y}(s), \quad s \in[0, T) .
$$


This gives another way of solving our LQ problem. Remark 5.6 tells us that the assumption of Theorem 6.6 is more general. As a special case when $Q \geq 0$ and $Q_{1} \geq 0$, we have

Corollary 6.7. If $Q \geq 0, Q_{1} \geq 0$ and $Q_{1}^{\frac{1}{2}} L_{T}$ is closed, then there exists a unique optimal pair $\bar{u}_{t, x}(\cdot), \bar{y}_{t, x}(\cdot)$ of Problem $(L Q)_{t}$ for any $x \in X$ and $t \in[0, T)$. Moreover, there exists a self-adjoint operator $P^{*}(t)=P(t)$, such that

(i) $P(\cdot) \in \mathcal{L}(X ; C([0, T] ; X))$;

(ii) In fact, even more, for $0 \leq \theta<1$,

$$
\left\|\left(A^{*}\right)^{\theta} P(t)\right\| \leq \frac{C_{\theta}}{|T-t|^{\theta}}
$$

(iii) For all $0<\varepsilon \leq T, 0 \leq \theta<1,\left(A^{*}\right)^{\theta} P(\cdot) \in \mathcal{L}(X ; C([0, T-\varepsilon] ; X))$;

(iv) For each $x \in X$ and $t \in[0, T)$, the optimal control $\bar{u}_{t, x}(\cdot)$ is given in pointwise feedback form by

$$
\bar{u}_{t, x}(s)=-R^{-1} B^{*}\left(A^{*}\right)^{\alpha} P(s) \bar{y}_{t, x}(s), \quad t \leq s<T
$$

$$
\langle P(t) x, x\rangle=J_{t, x}\left(\bar{u}_{t, x}(\cdot)\right)
$$

(vi) For $0 \leq t<T, P(t)$ satisfies the following Riccati differential equation, for all $x, y \in D\left(A^{\varepsilon}\right)$, for all $\varepsilon>0$,

$$
\begin{aligned}
\langle\dot{P}(t) x, y\rangle & =-\langle Q x, y\rangle+\langle P(t) A x, y\rangle+\langle P(t) A y, x\rangle \\
& +\left\langle R^{-1} B^{*}\left(A^{*}\right)^{\alpha} P(t) x, B^{*}\left(A^{*}\right)^{\alpha} P(t) y\right\rangle
\end{aligned}
$$

(vii) The following regularity properties hold for the optimal pair,

$$
\begin{aligned}
& \left\|\bar{u}_{t, x}(\cdot)\right\|_{\mathcal{U}[t, T]}+\left\|\bar{y}_{t, x}(\cdot)\right\|_{\mathcal{X}[t, T]} \leq c\|x\|, \\
& \left\|Q_{1}^{\frac{1}{2}} \bar{y}_{t, x}(T)\right\|_{X} \leq c\|x\|, \\
& \left\|\bar{u}_{t, x}(s)\right\| \leq \frac{c}{(T-s)^{\alpha}}\|x\|, \quad \forall s \in[0, T), \\
& \left\|\bar{y}_{t, x}(\cdot)\right\|_{C([t, T] ; X)} \leq c\|x\|, \quad \text { if } 0 \leq \alpha<\frac{1}{2} . \\
& \left\|\bar{y}_{t, x}(s)\right\| \leq \frac{\hat{c}_{\varepsilon}}{(T-s)^{2 \alpha-1-\varepsilon}}\|x\|, \quad \forall s \in[0, T), \quad \text { if } \frac{1}{2} \leq \alpha<1, \quad \varepsilon>0 ;
\end{aligned}
$$


(viii)

$$
\begin{gathered}
\lim _{t \rightarrow T} P(t) x=Q_{1} x ; \\
\lim _{t \rightarrow T}\left\langle Q_{1}^{\frac{1}{2}} \bar{y}_{t, x}(T), z\right\rangle=\left\langle Q_{1} x, z\right\rangle, \quad \forall z \in X ;
\end{gathered}
$$

where $c>0$ is an absolute constant but $C_{\theta}, \hat{c}_{\varepsilon}$ dependent of $\theta$ and $\varepsilon$ respectively.

Now, we give a simple explanatory example as an application of our results.

Example 6.8. Consider the one-dimensional heat equation with Dirichlet control action:

$$
\left\{\begin{array}{l}
\frac{\partial f}{\partial t}(z, t)=\frac{\partial^{2} f}{\partial z^{2}}(z, t), \quad 0 \leq z \leq 1, \quad t \geq 0 \\
f(0, t)=u(t), \quad f(1, t)=0 \\
f(z, 0)=f_{0}(z), \quad 0 \leq z \leq 1
\end{array}\right.
$$

where $f_{0}(\cdot) \in L^{2}(0,1)$. Define $X=L^{2}(0,1), U=\mathbb{R}$ and $-A=\frac{d^{2}}{d z^{2}}$ with $D(A)=H^{2} \cap H_{0}^{1}$. Both $X$ and $U$ are Hilbert spaces with the usual inner products. Let

$$
\lambda_{n}=n^{2} \pi^{2}, \quad \varphi_{n}(z)=\sqrt{2} \sin n \pi z, \quad \text { for } 0 \leq z \leq 1, \quad n=1,2, \cdots .
$$

Obviously, $\left\{\varphi_{n}(\cdot)\right\}_{n=1}^{+\infty}$ is an orthonormal basis of $X, A \varphi_{n}=\lambda_{n} \varphi_{n}$, and $-A$ generates an analytic semigroup $e^{-A t}$ on $X$, which is given by

$$
e^{-A t} \varphi(\cdot)=\sum_{n=1}^{+\infty}\left\langle\varphi, \varphi_{n}\right\rangle e^{-n^{2} \pi^{2} t} \varphi_{n}
$$

The fractional power $A^{\gamma}$ is well-defined $(\gamma \in \mathbb{R})$ :

$$
A^{\gamma} \varphi(\cdot)=\sum_{n=1}^{\infty}\left\langle\varphi, \varphi_{n}\right\rangle \lambda_{n}^{\gamma} \varphi_{n}, \quad \text { if } \sum_{n=1}^{+\infty}\left|\left\langle\varphi, \varphi_{n}\right\rangle \lambda_{n}^{\gamma}\right|^{2}<+\infty .
$$

Next, we consider the following problem

$$
\left\{\begin{array}{l}
\frac{\partial^{2} f}{\partial z^{2}}(z)=0, \quad 0 \leq z \leq 1 \\
f(0)=u, \quad f(1, t)=0
\end{array}\right.
$$


A straightforward calculation shows that the solution of (6.39) is

$$
G u=\sum_{n=1}^{+\infty} \frac{(-1)^{n} \sqrt{2}}{n \pi} u \varphi_{n}
$$

where $G$ is called the Dirichlet map of (6.39). For a fixed $\varepsilon \in\left(0, \frac{1}{4}\right)$, we denote $B=A^{\frac{1}{4}-\varepsilon} G$, which satisfies

$$
B u=\sum_{n=1}^{+\infty} \frac{(-1)^{n} \sqrt{2}}{(n \pi)^{\frac{1}{2}+2 \varepsilon}} u \varphi_{n} .
$$

Therefore, the mild solution of (6.36) is

$$
\begin{aligned}
f(z, t) & =e^{-A t} f_{0}(z)+\int_{0}^{t} A^{\frac{3}{4}+\varepsilon} e^{-A(t-s)} B u(s) d s \\
& =\sum_{n=1}^{+\infty}\left\{\left\langle f_{0}, \varphi_{n}\right\rangle e^{-n^{2} \pi^{2} t}+(-1)^{n} \sqrt{2} n \pi \int_{0}^{t} e^{-n^{2} \pi^{2}(t-s)} u(s) d s\right\} \varphi_{n} .
\end{aligned}
$$

Let $R=\left(1+2 \pi^{2}\right) I, Q_{1}=I-2 P_{1}$, where $P_{1}$ is given by

$$
P_{1} \varphi=\left\langle\varphi, \varphi_{1}\right\rangle \varphi_{1}, \quad \forall \varphi \in X,
$$

and

$$
Q \varphi=\sum_{n=1}^{+\infty}(-1)^{n}\left\langle\varphi, \varphi_{n}\right\rangle \varphi_{n}, \quad \varphi \in X .
$$

With the control system (6.36), we define the following quadratic cost functional over the time interval $[0,1]$ :

$$
J(u(\cdot))=\left\langle Q_{1} f(\cdot, 1), f(\cdot, 1)\right\rangle+\int_{0}^{1}\{\langle Q f(\cdot, s), f(\cdot, s)\rangle+\langle R u(s), u(s)\rangle\} d s .
$$

It should be pointed out that the state weight operators in (6.42) are indefinite. Obviously, we have

$$
D\left(L_{1}\right)=\left\{u(\cdot) \in L^{2}(0,1) \mid \sum_{n=1}^{+\infty} n^{2}\left[\int_{0}^{1} e^{-n^{2} \pi^{2}(1-s)} u(s) d s\right]^{2}<+\infty\right\},
$$




$$
\begin{aligned}
& \|L u(\cdot)\|_{L^{2}(0,1)}=\left\{\sum_{n=1}^{+\infty} 2 n^{2} \pi^{2} \int_{0}^{1}\left[\int_{0}^{t} e^{-n^{2} \pi^{2}(t-s)} u(s) d s\right]^{2} d t\right\}^{\frac{1}{2}} \\
& \leq\left\{\sum_{n=1}^{+\infty} 2 n^{2} \pi \int_{0}^{1}\left[\int_{0}^{t} e^{-n^{2} \pi^{2}(t-s)} d s \int_{0}^{t} e^{-n^{2} \pi^{2}(t-s)} u^{2}(s) d s\right] d t\right\}^{\frac{1}{2}} \\
& \leq\left\{2 \sum_{n=1}^{+\infty}\left[\int_{0}^{1} \int_{0}^{t} e^{-n^{2} \pi^{2}(t-s)} u^{2}(s) d s d t\right\}^{\frac{1}{2}} \leq \frac{1}{\sqrt{3}}\|u(\cdot)\|_{L^{2}(0,1)} .\right.
\end{aligned}
$$

Then

$$
\begin{gathered}
\left\langle\Phi_{0}(0) u(\cdot), u(\cdot)\right\rangle+\left\langle Q_{1} L_{1} u(\cdot), L_{1} u(\cdot)\right\rangle \geq \frac{2}{3}\|u(\cdot)\|_{L^{2}(0,1)}, \\
\forall u(\cdot) \in D\left(L_{1}(t)\right),
\end{gathered}
$$

which implies (5.18) holds for all $t \in[0,1)$ with $\delta=\frac{2}{3}$. Therefore, the assumptions of Theorem 5.5 or Theorem 6.6 hold. Although the state weight operators are indefinite and not smoothing, the synthesis problem of (6.36) with the quadratic cost functional (6.42) can be solved completely through our results.

Acknowledgment. The authors thank Professor Jiongmin Yong of Fudan University for many invaluable discussions and helps.

\section{REFERENCES}

[1] A.V.Balakrishnan, Boundary control of parabolic equation: $L-Q-R$ theory, in Non Linear Operators, Pro. 5th Internat. Summer School, Akademic-Berlin, 1978.

[2] A.Bensoussan, G.Da Prato, M.C.Delfour \& S.K.Mitter, Representation and Control of Infinite Dimensional Systems, Volume I, Systems \& Control: Foundations \& Applications, Birkhäuser, Boston Basel Berlin, 1992.

[3] A.Bensoussan, G.Da Prato, M.C.Delfour \& S.K.Mitter, Representation and Control of Infinite Dimensional Systems, Volume II, Systems \& Control: Foundations \& Applications, Birkhäuser, Boston Basel Berlin, 1993.

[4] S.Chen, Riccati equations arising in infinite dimensional optimal control problem, Control Theory Appl. 2 (1985), 64-72. (in Chinese)

[5] R.Curtain \& A.Pritchard, The infinite-dimensional Riccati equation for systems defined by evolution operators, SIAM J. Control and Optimiz. 14 (1976), 951-983.

[6] G. Da Prato, Quelques résultat d'existence unicité et régularité pour un problème de la théorie du contrôle, J. Maths. Pures Appl. 52 (1973), 353-375.

[7] G. Da Prato \& A.Ichikawa, Riccati equations with unbounded coefficients, Ann. Mat. Pura. Appl. 140 (1985), 209-221.

[8] F. Flandoli, Riccati equations arising in a boundary control problem with distributed parameters, SIAM J. Control and Optimiz. 22 (1985), 76-86. 
[9] F. Flandoli, A counterexample in the boundary control of parabolic systems, Appl. Math. Lett. 2 (1990), 47-50.

[10] F. Flandoli, On the direct solutions of Riccati equations arising in boundary control theory, Ann. Mat. Pura. Appl. CLXIII (1993), 93-131.

[11] A. Friedman, Partial Differential Equation of Parabolic Type, Prentice-Hall, Englewood Cliffs, New Jersey, 1964.

[12] T. Kato, Perturbation Theory for Linear Operators, Springer-Verlag, New York, 1980.

[13] I.Lasiecka, Unified theory for abstract parabolic boundary problem: a semigroup approach, Appl. Math. and Optimiz. 6 (1980), 283-333.

[14] I.Lasiecka \& R.Triggiani, Dirichlet boundary control problem for parabolic equations with quadratic cost: Analyticity and Riccati's feedback synthesis, SIAM J. Control and Optimiz. 21 (1983), 41-68.

[15] I.Lasiecka \& R.Triggiani, Differential and Algebraic Riccati Equations with Application to Boundary/ Point Control Problems: Continuous Theory and Approximation Theory, LNCS Vol.164, Springer-Verlag, New York, 1991.

[16] I.Lasiecka \& R.Triggiani, Riccati differential equations with unbounded coefficients and non-smooth terminal condition - The case of analytic semigroups, SIAM J. Math. Anal. 23 (1992), 449-481.

[17] X.Li \& J.Yong, Optimal Control Theory for Infinite Dimensional Systems, Systems \& Control: Foundations \& Applications, Birkhäuser, Boston Basel Berlin, 1995.

[18] J.L.Lions, Optimal Control of Systems Govened by Partial Differential Equations, Springer-Verlag, New York, 1971.

[19] B.P.Molinari, The Time-Invariant Linear-Quadratic Optimal Control Problem, Automatica 13 (1977), 347-357.

[20] L. Pan \& J. Yong, A differential game with multi-level of hierarchy, J. Math. Anal. Appl. 161 (1991), 522-544.

[21] A.Pazy, Semigroups of Linear Operators and Applications to Partial Differential Equations, Springer-Verlag, New York, 1983.

[22] Y.You, Optimal control for linear systems with quadratic indefinite criterion on Hilbert spaces, Chin. Ann. Math. Ser.B 4 (1983), 21-32.

[23] Y.You, On the solution of a class of operator Riccati equations, Chin. Ann. Math. Ser.A 5 (1984), 219-227. (in Chinese)

[24] Y.You, Closed-loop optimal solution to quadratic boundary control of parabolic systems, Acta Math. Sinica 28 (1985), 809-816. (in Chinese) 